Aus dem anatomisch-biologischen Institut der Universitït Berlin.

\title{
Über die Genese des Chordaknorpels der Urodelen und die Natur des Chordagewebes.
}

\author{
Von
}

Dr. med. Friedrich Krauss in Charlottenburg.

Hierzu Tafel IV--VI.

Trotz der reichlich vorhandenen Literatur über die Struktur and Natur der Chorda differieren doch noch die Ansichten der Autoren über die Genese des Chordaknorpels; auch fehlt es noch an Arbeiten, welche die feineren, histologischen Details mit Zuthilfenahme der heutigen verbesserten Untersuchungsmethoden beriicksichtigen. Es hat aber eine nicht geringe Bedeutung, dieses Thema in erschöpfender Weise zu behandeln und zu einer einheitlichen Anschauung in betreff desselben zu gelangen, weil die Frage nach der Bildung des Chordaknorpels aufs innigste mit der Naturder Chorda, dieses für uns noch so manches Ratselhafte bietenden Gewebes, zusammenhungt. In der Tat werden wir finden, wenn wir die Literatur über die Arbeiten durchsehen, welche sich mit dem Chordaknorpel beschaftigen, dass die betreffenden Verfasser sich in ihren Anschauungen dabei mehr oder weniger von ihren Ansichten über die Beschaffenheit und Entwicklung des Chordagewebes beeintlussen liessen oder umgekehrt auch von dem Resultate ihrer Untersuchungen über den Chordaknorpel ihre Auffassung des Chordagewebes und seine Stellung in der Reihe der Gewebsformationen abhăngig machten.

Weiterhin ist aber das Thema von der Herkunft des Chordaknorpels von Bedeutung für die Frage nach dem Wesen und der Herkunft der Stützgewebe. Hat doch gerade die Auffassung dieser Frage viel zu der Stellungnahme beigetragen, welche man in neuerer Zeit der Bedeutung der alten Keimblätterlebre für die Entstehung und Abgrenzung der Gewebe entgegenbringt. Eine nicht minder wichtige Rolle spielt, wie wir im Verlaufe der Arbeit zeigen werden, bei der Bildung des Chordaknorpels auch die Metaplasie der Gewebe, eine Frage, die heute im Vordergrund des Interesses steht. 


\section{Historisches.}

In diesem Abschnitt soll nur die speziellere Literatur des Chordaknorpels besprochen werden; die der Chorda selbst, welche überaus reichbaltig ist, wird soweit erforderlich, gelegentlich der allgemeinen Besprechung berücksichtigt werden. Eine sehr eingehende Aufstellung derselben findet sich übrigens bei $\mathrm{H}$. Schauinsland: die Entwicklung der Wirbelsänle nebst Rippen- und Brustbein. (O. H e r twig, Handbuch der Entwicklungslehre III, Teil 2.)

Nachdem v. Kölliker 1859 und 1860 bei Knochenganoiden, bei Polypterus und Lepidosteus, auf das Vorkommen von Kinorpelgewebe am kaudalen Ende der Chorda hingewiesen hatte, hat Gegen ba ur in seiner klassischen Monographie: Untersuchungen zur vergleichenden Anatomie der Wirbelsăule bei Amphibien und Reptilien, Leipzig 1862 , bei einer grossen Reihe von Tierklassen den Chordaknorpel in eingehender, noch heute mustergältigel Weise beschrieben und auch die feineren histologischen Verhaltnisse in einer im Verhältnis zu der damals noch primitiven Technik bewundernswerten Weise klargelegt. ( $\mathrm{e}$ g e $n$ b a u $r$ fand den Chordaknorpel in erster Linie bei lirodelen und Gymnophionen, dann bei einzelnen Reptilien und Vögeln, sowie ihm ahnliche Gebilde auch bei Knochentischen. Nach Gegenbaur entsteht der Chordaknorpel innerhalb der Chorda aus den peripherer Chordapartien. Gegenbaur hatte in seiner Arbeit bereits ein reiches Tatsachenmaterial zur Behandlung der Frage beigebracht. Die zahlreichen ibm folgenden Bearbeiter des Gegenstandes haben in morphologischer Hinsicht nicht viel Neues himzuzufügen vermocht. Die seit Gegenbaur bis gegen 1900 erschienenen Arbeiten (es sind dies die letzten) beziehen sich vorwiegend auf die $\mathrm{Ent}$ s te hu ng des Chordaknorpels.

Wăhrend ein Teil der Autoren den Ursprung des Chordaknorpels von einer Einwanderung von Knorpelzellen aus dem perichordalen Gewebe, speziell dem Intervertebralknorpel, durch Öffnungen der Elasitca externa herleitet, halt dagegen ein anderer Teil und zwar die Mehrzahl der heutigen Forscher, an der Entstehung aus der Chorda selbst und besonders aus dem Chordaepithel fest. Daneben gibt es einige Forscher, welche an beide genannte Entstehungsmöglichkeiten glauben. 
Von den Vertretern der ersteren Ansicht, der perichordalen Entstehung, ist in erster Linie Zyk off (1894) zu nennen, welcher energisch die alte Gege nbaursche Ansicht bekämpfend annahm, dass ein Teil der Zellen des Intervertebralknorpels, sich zwischen die Chordascheide und die Wandung des Wirbels zum Zentrum des Wirbels drängend, in die Chorda eindringe. Die Möglichkeit solchen Eindringens erklärt Zy k off sich dadurch, dass die Elastica externa bei dem Siredon durchlöchert, stellenweise zerrissen sei. Ich bemerke schon hier, dass die Präparate Zy k offs in technischer Beziehung voll rerschiedener Seite als nicht einwandsfrei angesehen wurden.

Auch nach L w off (1887) wird der Knorpel, welcher sich in der Chorda befindet, nicht ans den Elementen der Chorda gebildet, sondern wächst aus dem perichordalen Gewebe hinein. In der Elastica externa sind öffnungen $z u$ sehen, in welchen man sehr oft an gefärbten Präparaten gefarbte Körper, oftenbar Zellen des Bindegewebes, sieht. Sowohl $I, w$ of $f$ als $Z y$ k of $f$ wurden in ihren Ansichten über die Entstehnngsweise des Chordaknorpels bestarkt durch ihre Auffassung von der Natur der Chorda als eines epithelialen Gewebes.

Eine ahnliche Ansicht, wie die der beiden genannten Forscher, wurde von Gadow (1896) ausgesprochen, welcher, olne selbst genauere histologische Untersuchungen ansgeführt zu haben, die Bildung des Chordaknorpels bei den Urodelen mit der Knorpelbildung der Chorda der Selachier in eine Parallele bringt.

Schliesslich führe ich von Vertretern dieser Riclitung noch Studnička (1897) an, welcher in sehr ausführlicher Weise über die Chorda und den Chordaknorpel geschrieben hat und dabei auch die Histogenese des Knorpelgewebes behandelt. Studnička ist ein entschiedener Anbänger der perichordalen Entstehungsweise des Chordaknorpels und gibt nur für eine geringe Anzahl von Fällen eine Entstehung aus den Chordaepithelzellen zu. Bestimmenden Eintluss hat dabei seine Ansicht von der epithelialen Natur der Chorda, zu der er sich lediglich durch die Existenz interzellulärer Verbindungen in der Chorda der Knochenfische bestimmen lässt, trotzdem er nicht in Abrede stellt, bei verschiedenen Gelegenheiten Bilder der Chorda angetroffen zu haben, welche eine grosse Ähnlichleit mit Knorpelgewebe haben. 
Was nun die Entstehung des Chordaknorpels a us der Chorda selbst betrifft, so kann man sagen, dass die Mehrzahl der neueren Autoren sich jetzt diesem Bildungsmodus, dem endochordalen, mehr oder weniger zuneigt.

Zunächst ist hier V. Sc h midt (1893) zu nennen. $\mathrm{Er}$ studierte die Chordastabbildung in der Schwanzspitze des Axolotl und fand, dass sich hier Knorpel direkt aus Chordazellen bildete und zwar aus den hier gewöhnlich noch indifferenten protoplasmatischen Zellen des äusseren Chordaendes. Dabei beobachtete er gleichzeitiges Auftreten von hyalinen Tropfen in den Chordazellen als Anfang ibrer hyalinen Umwandlung zu Knorpelgewebe. Für eine Einwanderung von Zellen aus der skelettogenen Schicht in den Chordastab fehlt nach ihm jeder Anhalt, da um die Zeit der Gliederung des Chordastabes eine solche Schicht im Bereich des Chordaendes noch garnicht vorhanden ist.

Alsdann fand Field (1895) bei Amphiuma, dass die Chordazellen und zwar speziell diejenigen des Chordaepithels, die Elemente für den Knorpel bilden. Die Chordascheide fand er stets als vollständige Hülle um die Chorda gehend und nirgends die von $\mathrm{Zyk}$ off beschriebenen Unterbrechungen. Auch eine Durchwucherung der intakten Chordascheiden durch skelettbildende Zellen hält Field für durchaus unwahrscheinlich.

Auch v. Ebner (1896) erklärt wie Field eine primäre Knorpelbildung, d. h. eine Umwandlung der Chordaepithelzellen in Knorpel in der Schädelchorda der Salamander- und Tritonenlarven für zweifellos, wenn er auch die Zy k of fschen Ansichten nicht ganz von der Hand weist. Auch gibt v. Ebner an, dass später oft eine teilweise \%erstörung der Chordascheide und dadurch ein Zusammentliessen des Parachordalknorpels mit dem Chordaknorpel eintrete. v. Ebner halt die Chorda, besonders die der Knochenfische, für ein hochdifferenziertes Gebilde, welches oft die mannigfachsten progressiven Bildungen, wie z. B. bindegewebige Scheiden, Knorpel, vakuolisierte Zellen, epidermoidale Zellen produziert und als ein Beispiel dienen kann, wie vergeblich das Bemühen sei, einen fundamentalen Gegensatz zwischen Bindeund Epithelgewebe aufzustellen.

Ferner tritt Klaatsch (1897) sehr entschieden für die ältere Gegenbaursche Anschauung von der Lintstehung des Chordaknorpels aus der Chorda ein. Klats ch fand neben 
peripheren Knorpelinseln auch zentrale und neigt zu der Ansicht, dass letztere vielleicht unabhängig von ersteren entstanden seien. Die Bildung des Chordaknorpels geht nach Klatsch in erster Linie von den indifferenten Chordaepithelzellen aus und zwar findet als erste zur Knorpelbildung führende Veränderung eine Verdickung der peripheren Zellpartien des Chordaepithels statt, in welchen eine durch Hämatoxylin dunkler tingierbare, teils in Form von Streifen, teits in Form eines Netzwerkes hervortretende Substanz sich bildet. Für die zentralen Knorpelinseln gibt Kilaatsch auch die Vöglichkeit $z u$, dass sie aus mehr differenten, aber noch wenig vakuolisierten Chordazellen entstehen können. Klatsch neigt zu der Ansicht, dass Chorda- und Knorpelgewebe aufs innigste miteinander verwandt seien, vielleicht nur als Zweige eines und desselben Stammes eines indifferenten Stützgewebes aufzufassen seien. Klatseh trug zunachst seine Ansichten in der Anatomischen Gesellschaft 1897 vor. In der sich daran knüptenden Diskussion prïzisierte $\mathrm{Sch}$ af fer entsprechend seinen in mehreven umfangreichen Arbeiten über die Entstehung des Knorpelgewebes niedergelegten Ansichten seinen Standpunkt dahin, dass der Chordaknorpel sich nur aus Chordaepitbelzellen, welche den Wert von indifferenten, embryonalen Zellen haben, nicht aus schon vakuolisierten entwickeln könne. Daneben glaubt aber Schaffer auch noch an gelegentliche Entstehung von Knorpel aus eingewanderten indifferenten Zellen (Chondroblasten) und berief sich dabei ausser auf seine bei Petromyzon gemachten Beobachtungen über Knorpelwucherungen auf die v. Ebnerschen Lintersuchungen.

Von den jüngeren Autoren, welche über den Chordaknorpel geschrieben haben, ist Ka pelkin (1900) zu nenuen. Ka pelkin meint, dass die Schwierigkeit, die Frage der Fntstehung des Chordaknorpels zu lösen, vielfach daran gelegen ware, dass in einem gewissen Stadium sich die Chordascheiden etwas auflösen und deswegen sich wenig von der Grundsubstanz des Knorpels durch ihren Lichtglanz unterscheiden. Durch zweckmässige Doppelfärbung überwand er diese Schwierigkeit und gelangte zu dem Resultat, dass der Chordaknorpel sich auf Kosten des Chordaepithels ganz unabhängig vom Intervertebralknorpel entwickie.

Schliesslich ist noch Camillo Schneider anzuführen, welcher in seinem Lehrbuch der Histologie der Tiere, Jena 1902, 
eingehende Details über die Bildung des Chordaknorpels beim Salamander gibt. Nach ihm nehmen nur die Chordaepithelzellen an der Knorpelbildung teil und von diesen auch nur ein Teil, während die anderen degenerieren. Nur die wandständigen Zellen des Chordaepithels liefern echten hyalinen Knorpel, wăhrend die durch Teilung entstandenen und ausgewanderten ein eigentümliches Gewebe bilden, welches aus kleineren Zellen und einer lockerfaserigen, schwach chondromukoiden Grundsubstanz besteht. Dieses Gewebe soll sich nicht immer zu echtem Knorpel fortentwickeln, denn man kann Reste desselben im. Zentrum des Chordaknorpels in der Umgebung der geschrumpften Chordazellen wahrnehmen.

\section{Untersuchungsgebiete, Material und Technik.}

Meine Untersuchungen über Chordaknorpel wurden hauptsächlich an Urodelen: Siredon pisciformis, Salamandra maculata und Triton cristatus (Larven sowohl, als auch erwachsenen Tieren) in verschiedenen Stadien vorgenommen. In erster Linie erwies sich der Axolotl ais geeignetes Untersuchungsobjekt, besonders für die feineren histologischen Verhaltnisse der Chorda und ihrer Verknorpelung, weil hier die zelligen Elemente am grössten sind und auch bei der weiteren Umbildung der Wirbelsäule wïhrend des späteren Wachstums die Verbăltnisse sich weniger komplizieren als beim Salamander und Triton, bei denen die Chordazellen kleiner sind und die Verknorpelungsprozesse unregelmässigere Verbreitung zeigen. Immerhin sind aber auch bei den letztgenannten Tieren bemerkenswerte Eigentümlichkeiten in den histologischen Details gefunden worden. Zur Ergänzung und Vervollständigung der Untersuchung wurde dann noch die Chorda des Hechtes (Esox lucius) untersucht und zwar an einem erwachsenen Exemplar von $2 \overline{\mathrm{cm}}$, da jüngere Tiere nicht zu beschaffen waren. Ferner wurden bei Axolotl- und Forellenembryonen die Verhältnisse der Chordaneubildung bei der Regeneration des Schwanzes nach Amputation desselben in verschiedenen Stadien untersucht, daneben auch die Folgen der Kontinuitätstrennung der Chorda. Schliesslich wurden mehrfache Transplantationen der Chorda von meist $3 \mathrm{~cm}$ langen Axolotllarven unter die Rackenhaut erwachsener Axolotl vorgenommen und das Schicksal der transplantierten Chorda nach verschiedenen Zeitabschnitten studiert. 
Die Objekte wurden zumeist in Pikrinsublimatessigsäure, zuweilen auch in Carnoys oder in Flemmings Gemisch, fixiert. Letztere Flüssigkeit, welche so ausgezeichnet die feinen Zellstrukturen konserviert, hat den Nachteil, dass sie keine genügende Knorpelfărbung zulässt.

Die Einbettung geschah in Paraffin.

Zum Studium des Chordaknorpels wurden Frontal- und Que1schnitte, zuweilen auch Sagittalschnitte, angefertigt: meist Serien kleinerer Abschnitte. Was die Frontalschnitte anbetrifft, so ist eine vorgängige gehörige Orientierung an dem Paraffinstücke wichtig, was besonders bei den meist kleinen Objekten nicht immer leicht ist. Ich habe dazu zuerst von beiden Seiten des Blockes Querschnitte gemacht, dann dieselben mikroskopisch untersucht, sodass ich die Stelle der Chorda auf der glatten Querschnitttäche genau wiederfinden und durch eine dieselbe schneidende, horizontale Linie mit dem Skalpell markieren konnte. Ich habe dann die Linien der beiden Querschnitte noch seitlich verbunden und so stets die richtige Schnittebene erhalten.

Die auf dem Objektträger aufgeklebten Schnitte wurden mit verschiedenen Farbungen behandelt. Die besten Dienste leistete mir das Kresylviolett RR (Farbwerke Mülheim, vormals d. Leonhard \& Co.), welches hervorragende Eigenschaften als Knorpelfarbemittel besitzt. Das Kiresylviolett gehört zu den metachromatisch farbenden Farbstoffen. Es fürbt die Finorpelgrundsubstanz, sowie auch das Mucin rosarot, die Kerne hellblau. Ich fand es äusserst vorteilhaft, eine Färbung mit Hämalaun voranszuschicken, da die Kernfarbung des Hämalauns besser haltbar ist und ihr Blan besser zu dem Rot des hiesylvioletts liontrastiert. Der Alkohol zieht nämlich das Kresylviolett leicht aus den Präparaten heraus, deshalb muss auch die Durchführung derselben durch die verschiedenen Alkoholstufen eine sehr schnelle sein; auch muss durch mehrmals gewechseltes Xylol der Alkohol gründlich entfernt werden, bevor man die Präparate in Kanadabalsam einlegt. Derart behandelte Präparate haben sich mir bereits über ein Jahr lang gehalten, ohne dass sie wesentlich gelitten hätten. Es ist deshalb auch nicht nötig, was empfohlen wird, die Prăparate zur Vermeidung der Alkoholbehandlung in Lävulose einzulegen. $\mathrm{Zu}$ bedauern ist nur, dass der Farbstoff jetzt aus dem Handel verschwunden ist, wenigstens sind die 
unter anderen Marken jetzt kursierenden Präparate für die Knnorpelfárbung nicht brauchbar.

Als weitere Knorpelfärbemittel habe ich Methylenblau und B ismarckbran angewandt und zwar das Methylenblau in der von Hansen angegebenen Form mit einem kleinen Zusatz von Salzsaiure. Ich habe die Methylenblaufärbung mit molybdannsaurem Ammoniak fixiert und dann zuweilen noch die van Giesonsche, von $\mathrm{Ha}$ usen modifizierte Bindegewebsfärbung nacifolgen lassen. 1)as Bismarckbraun wird sehr gut mit Lichtgrün kombiniert. Sehr hübsche Bilder gibt eine dreifache lairbung in der Reiluenfolge: Boraxkarmin, Bismarckbraun (ganz schwach) und Lichtgrün. Man erhält dann die Kerne rot, die Knorpelgrundsubstanz braun, das Bindegewebe grün, andere Teile, wie Muskulatur, in einer mehr grauen Mischfarbe. Zur Kontrolle habe ich vielfach das gleiche Präparat mit den genannten verscliedenen Knorpelfurbemitteln behandelt.

Von sonstigen Fürbungen wurden noch die van Giesonsche nnd die Heidenhainsche mit Eisenalaun-Hümatoxylin, letzteres besonders für die in Flemmingscher Lösung fixierten Objekte, angewandt.

Auch wurde von der Doppelfärbung $\mathrm{H}$ ämala un-Eosin öfters Gebrauch gemacht und zwar letyteres in progressiver Farbung (einige Tropfen konzentriert wässeriger Eosinlösung auf ein Farbglas Aqua destillata: 24 Stunden lang färben).

\section{Eigene Befunde.}

I. Die Bildung des Chordaknorpets bei den Erodelen.

Wir werden uns bei der Schilderung des chondrogenetischen Prozesses, wenn nicht besonders hervorgehoben, an die Axolotllarve lalten, da beim Salamander und T'riton die Vorgänge, wenn auch im wesentlichen dieselben, doch weniger durchsichtig sind, weil hier die Zellelemente lileiner und die Verknorpelung eine unregelmüssigere ist.

Beim Studium der Bildung des Chordaknoryels hat sich mir rnehr und mehr als wichtig herausgestellt, die Vorgänge am Chordaepithel von denjenigen, welche sich an den vakuolisierten Zellen abspielen, als gesondert zu betrachten. Von letzteren hatte man bisher angenommen, dass sie sich am Verknorpelungsprozess wenig oder gar nicht beteiligten und 
durch das vom Chordaepithel neugebildete Knorpelgewebe zerstört würden. Diese Ansicht erschien mir im Verlauf meiner Untersuchungen mehr und mehr zweifelhaft und so richtete ich im Hinblick darauf, dass beim Axolotl sich der Chordaknorpel im Vertebralteil der Chorda entwickelt, während der Intervertebralteil frei von Veränderungen bleibt, mein Augenmerk darauf, ob nicht schon in einem ganz frühen Stadium morphologische Unterschiede im Verhalten der vakuolisierten Zellen des Vertebralteils im Vergleich zu denjenigen des Intervertebralteils der Chorda zu finden seien. Und so zeigten sich bereits bei Axolotllarven von $3^{1 / 2} \mathrm{~cm}$ Länge Veränderunge! in der Struktur der Chordazellen, welche nur auf den Vertebralteil der Chorda beschrankt sind und als erste Phase der Bildung des Chordaknorpels, als Einleitung des chondrogenetischer Prozesses, aufgefasst werden müssen (s. Fig. 1, Taf. IV). Diese auf die genannten Stellen beschrïnkten Veründerungen bestehen im Auftreten eines fädigen Netzwerkes im flüssigen Vakuoleninhalt der Chordazellen (Fig. 2, Taf. V). Die Füden des Netzwerkes ziehen vielfach verzweigt vom Proto- oder Endoplasma der Zellen zur Vakuolenwand oder endigen frei in der Flüssigkeit. Die Fäden scheinen mir nur zum Teil als protoplasmatische Ausläufer des Endoplasmas der vakuolisierten Zellen aufzufassen zu sein. Grösstenteils dürften sie, wie weiter unten noch besprochen werden wird, eine andere Herkunft haben. Mit Farbstoffen, wie Hämatoxylin, Bismarckbraun, Kresylviolett nehmer die Fäden häufig dieselbe Färbung an wie das Chondromukoid '). Auch konnte ich öfters beobachten, dass die Faden anfänglich aus kleinsten, rosenkranzartig aneinander gereihten'Tröpfchen bestehen, welche später zu linearen Faden verschmelzen und an welchen zuweilen noch kleine Verdickungen als Ausdruck ihrer früheren

1) Ich möchte an dieser Stelle bemerken, dass hier sowohl, wie im weiteren Verlauf der Arbeit, wo von chondromukoider Umwandlung die Rede ist, ich im Sinne Hansens darunter die Eigenschaft des Gewebes verstehe, mit den genannten Farbstoffen die charakteristische Knorpelfärbung anzunehmen, ohne Rücksicht darauf zu nehmen, dass das Chondromukoid sich mit dem Mucin färberisch gleich verhält. Ein sicheres färberisches Reagens, welches beide Substanzen auseinander zu halten ermöglicht, gibt es nicht. Auch das Hämalaun (S chaffer [41]) ist hierfür nicht zuverlässig. Viel hängt dabei von dem Alaungehalt und der vorherigen Art der Fixation des Objektes ab. 
Entstehungsweise aus Tröpfchen oder Kügelchen zu beobachten sind. Dieses Stadium der füdigen Struktur der vakuolisierten Chordazellen findet sich nun zu einer Zeit, zu welcher am Chordaepithel keine Spur einer Veründerung zu bemerken ist und ich halte es für wichtig, dieses Verhalten gleich anfangs zu betonen, da ich in demselben einen wichtigen Beweis für die hervorragende Anteilnahme der vakuolisierten Chordazellen am Verknorpelungsprozesse der Chorda erblicke. Soviel ich ersehen konnte, findet sich in früheren Arbeiten keine derartige Angabe verzeichnet. Wohl wurde beobachtet, dass zuweilen Fäden in den Vakuolen der Chordazellen vorkommen, aber es ist nicht auf die dabei auftretende chondromukoide Beschaffenheit und auf das alternierende Vorkommen dieser Struktur in den einzelnen Chordaabshnitten und zumal zu einer Zeit geachtet worden, in der sich die Bildung des Chordaknorpels vorbereitet.

Gleichzeitig oder etwas später als die fïdigen Strukturen treten nun auch Verdickungen der Membran der Chordazellen auf (Fig. 2, Taf. IV). Diese verdickten Scheidewünde haben auf dem Durchschnitt ein trübes, wie gequollenes Aussehen. Es scheint sich dabei ebenso wie bei einem 'Teil der fádigen Strukturen um ein Ausscheidungsprodukt des Zellprotoplasmas, d. h. um eine albuminoide Substanz zu handeln, welche mit dem Chondromukoid nahe verwandt ist und der chondromukoiden Metamorphose vorausgeht. Nach meinen Beobachtungen möchte ich annehmen, dass das Chondromukoid überhaupt entweder direkt als solches ausgeschieden wird oder zuerst als die vorhin genannte, nicht farbbare albuminoide Substanz, welche erst spater vielleicht nur durch eine gerinfügige, chemische Umwandlung die charakteristische Chondromukoidfarbung annimmt. Fürberisch konnte ich keinen wesentlichen Unterschied $z$ wischen diesen verdickten und den nicht verdickten Membranen konstatieren. Die Verdickung der Chordazellmembran kann nun bereits in dieser frühen Zeit eine ziemlich betrïchtliche sein, auch kann es frühzeitig in den verdickten Membranpartien zu einer Umwandlung im Chondromukoid oder zu einer Ausscheidung desselben kommen; letzteres meist in Form von Tröpfchen oder von verschiedenartigen Figuren, welche durch das Zusammenfliessen von Tröpfchen entstanden sind. 
Erst nachdem so die Chondrogenese an den vakuolisierten Chordazellen eingeleitet worden ist, treten nun gewöhnlich die Veränderungen am Chordaepithel auf. Zum Verständnis der an demselben und ihrer Umgebung sich abspielenden Prozesse ist es von Wichtigkeit, das Chordaepithel and die an dasselbe grenzende Faserscheide der Chorda als eine einheitliche zus ammengehörige Gewebsbildung aufzufassen. Nach den Untersuchungen von v. Ebner wissen wir, dass entwicklungsgeschichtlich von den peripheren Chordazellen zuerst die Elastica externa und hierauf die Faserscheide gebildet wird. v. Ebner hält die Faserscheide für eine bindegewebige, dem Gallertgewebe nahestehende Gewebsbildung. Auch anatomisch ist das Chordaepithel innig mit der kaserscheide verbunden. Die Substanz des Protoplasmas der Chordiepithelzellen geht häufig direkt in die Fasern des Scheidengewebes über. Andererseits liegt die Chordaepithelzelle nach der Chorda hin häutig so, dass sie in den Zwischenraum zweier aneinanderstossender vakuolisierter Zellen keilförmig sich hineinschiebt und bei Wucherungsund Vermehrungsprozessen die Iembranen der vakuolisierten Zellen leicht auseinanderdrängen kann. Der chondrogenetische Prozess, der nun durch die Chordapithelzellen, welche ausserst bildungsfähig sind und an embryonale \%ellen erinnern, eingeleitet wird, kann als ein äbnlicher Prozess, gewissermaßen als eine weitere Fortsetzung des entwicklungsgeschichtlichen betrachtet werdell. Xan kann wohl schon im ersten beginn des Prozesses Teilung und Vermehrung der Chordaepithelzellen wahrnehmen: das wesentliche ist aber die Bildung eines protoplasmatischen Netzwerkes (Fig. 5, Taf. V; Fig. 3, Taf. IV) zwischen Chordaepithelzellen und Faserscheide, wodurch erstere von letzterer abgehoben werden. Es entsteht auf diese Weise ein kleiner, nach der Aitte der Chorda zu vorspringender Wulst (Fig. 6, Taf. V), welcher bei weiterem Wachstum eine mehr homogene Beschaffenheit annimmt und die ursprüngliche Chordaepithelzelle umschliessen und einspinnen kann. Dieser Vorgang findet, wie bereits Ka pelk in hervorgehoben hat, anfangs nur an einigen Stellen der Peripherie derChordazellen statt. Bemerkenswert ist, dass eine'Teilung und Vermehrung des Chordaepithels nunim Anfang nicht immer zu konstatieren ist; es kann vielmehrder erste Anfang des Prozesses ohne Epithelvermehrung vor sich gehen. 
Sehr frübzeitig kann eine chondromukoide Ümwandlung derwulstförmigen Bildung eintreten und gleichzeitig eine solche Verschmelzung mit der Faserscheide, dass man keine Grenze mehr zwischen beiden unterscheiden kann. Man hat dann eine bis zur Elastica externa sich gleichmässig färbende Masse vor sich. Häufig kommt es zwischen Wulst und Faserscheide zur Bildung kleiner Vakuolen; zuweilen bilden dieselben reihenförmig angeordnet die Grenze zwischen Chordaknorpel und Faserscheide. Sie entstehen entweder durch erweiterte Maschen des protoplasmatisclien Netzwerkes oder durch stärkere Abhebung desselben von der Faserscheide.

Während des Wachstums des Wulstes hat sich auch das Chordaepithel in seiner Nachbarschaft in lebhafter Weise vermehrt und zwar, wie mir scheint, mehr passiv durch den Reiz, welchen der gewissermaßen als Fremdkörper wirkende kompakte Wulst auf die Umgebung ausübt. Die jungen Zellen wandern alsdann wie Vorläufer an die Peripherie des Wulstes (Fig. 6, Taf. V), wo sie meist frübzeitig in die sich dort bildende Grundsubstanz eingeschlossen und zu Knorpelzellen werden. Es ist aber auch möglich, dass sie vorher die in den vakuolisierten Chordazellen vor sich gehenden metaplastischen Prozesse beeinflussen. Auch können sie, wie besonders beim Salamander, direkt zur Bildung neuen Knorpelgewebes beitragen. Nach der Vermehrung und Auswanderung der Chordaepithelzellen können oft grosse Strecken des inneren Randes der Faserscheide frei von solchen bleiben.

Im weiteren Verlauf des chondrogenetischen Prozesses kann der Knorpelwulst nun mit anderen ähnlichen peripherischen Wülsten verschmelzen oder auch mit dem von den vakuolisierten Chordazellen gebildeten fädigen Netzwerk sich verbinden und können so allmählich umfängliche Partien der Chorda zur Verknorpelung kommen.

Nachdem nun die Bildung des Chordaknorpels von seiten des Chordaepithels in der beschriebenen Weise in die Wege geleitet worden ist, werden unterdessen auch die vakuolisierten Chordazellen, in welchen es bisher nur zu Membranverdickungen und fädigen Netzbildungen im Innern der Vakuolen gekommen war, mehr und mehr in Mitleidenschaft gezogen. Das Endoplasma der vakuolisierten Zellen gewinnt im Verhăltnis zu dem Exoplasma, den Vakuolenwänden, welche früher ein integrierender 
Bestandteil der Gesamtzellen waren, eine grössere Selbständigkeit und kann nunmehr für sich als Zelle betrachtet werden, während die verdickten Vakuolenwände mehr und mehr sich von der eigentlichen Zelle absondern und interzelluläre Natur annehmen. Die anfänglichen Veränderungen an den Vakuolenwänden nehmen nun $z u$ und aus den verdickten Membranen entstehen dicke, faserreiche Balken. Die Fasern der Balken ziehen meist parallel zueinander. Das Netzwerk im Innern der Vakuolen wird dichter, auch können innerhalb der zu Balken verdickten Scheidewände netzförmige Züge auftreten, welche sich teils aus komprimierten mit Netzwerk erfüllten Vakuolen, grösstenteils aber mehr selbständig zwischen den auseinandergewichenen Faserzügen der Scheidewände gebildet haben (Fig. 9, Taf. IV; Fig. 4, 6, 'Taf. V).

Alle diese in und $z$ wischen den vakuolisierten Zellen sich vorfindenden faserigen, netzförmigen und membranösen Bildungen zeichnen sich durch ein von echtem Protoplasma verschiedenes Verhalten aus. Wenn auch durch Farbemittel kein Unterschied nachzuweisen ist, so spricht, abgesehen von der später zu besprechenden Art ihrer Genese, die trübe, graue Farbe und die vielfach wie gequollene Beschaffenheit dieser Gewebe dafür, dass sie rom Protoplasma verschieden sind. Ich gebe aber zu, dass es bei den feineren Făden nicht immer möglich ist, \%u sagen, wie weit das Endoplasma der Zellen reicht und wo diese mehr interzellularen Charakter tragenden Bildungen anfangen.

An der Hand der auf Tafel VI abgebildeten schematischen Figuren wollen wir die Details dieser Bildungen näber erörtern. Die Vakuolen zeigen die verschiedensten Grössen und Formen, vielfach sind sie grösser als die gewöhnlichen Chordavakuolen, oft über das Doppelte; es gibt aber auch kleinere. Wie ich glaube, sind die grösseren durch Steigerung des Flüssigkeitsdruckes im Innern der Vakuolen bei der Kompression des Gewebes durch den andrăngenden Chordaknorpel und durch Schwund von benachbarten Wänden entstanden, während die kleineren teils durch Schrumpfung der Wände mit Flüssigkeitsverlust, teils durch Faserbildung im Innern der Vakuolen sich gebildet baben. Die Form der Vakuolen ist ebenfalls sehr verschieden. Die Kerne liegen băufig nicht am Rande, sondern in der Mitte der Vakuolen (Fig. 40, Taf. VI), zuweilen auch in den verbreiterten Scheidewänden oder in den durch Zusammenstoss derselben gebildeten 
Zwickeln und sind oft sehr chromatinreich (Fig. 6, Taf. V; Fig. 2S, 42, 44, 45, Taf. VI). In seltenen Fällen konnte ich zwei Kerne in einer Vakuole wahrnehmen (Fig. 31, Taf. VI). Sehr interessant ist der Inhalt der Vakuolen. Oft geht von einem in der Mitte der Vakuolen gelegenen Kern, welchen eine geringe Menge Protoplasmas umgibt, ein meist radiär verlaufendes Faserwerk aus (Fig. 40, Taf. VI). Häufig trifft man Vaknolen ohne Kern, welche nur von Faserwerk erfüllt sind oder von quergespannten Fìden durchzogen werden (Fig.9, Taf. IV; Fig. ̄, Taf. V; Fig. 32,36, Taf. VI; Fig. 14, Taf. IV); ferner kommen als Vakuoleninhalt bäufig zarte Membranen (Fig. 29, 31, 32, 33, Taf. VI) oder unregelmässig umrandete Teile derselben vor, oft schleierartig ausgespannt (Fig. 29, 33, 34, 'Taf. VI) oder mehr zusammengewunden (Fig. 30, Taf. VI). Alle diese Gebilde können in der mannigfachsten Weise durch Faden und Faserwerk sich mit der Vakuolenwand verbinden oder auch mehr frei in der Vakuolenflüssigkeit suspendiert sein. In letzterem Falle sieht man sie hăufig fein baumförmig verzweigt endigen (Fig. 26-28, Taf. VI). Die Fasern selbst sind von verschiedener Dicke, meist selrr fein und zart, können sie sich so verdicken, dass sie breite Septen innerhalb der Vakuolen bilden (Fig. 4, Taf. V). An allen genannten Bildungen treten früher oder später chondromukoide Umwandlungen auf. Meist in Form von Tröpfchen, einzeln oder zu Fäden aneinander gereiht (Fig. 25-28, Taf. VI), oft auch in Form grösserer Tropfen oder mannigfaltigel eigentümlicher Figuren. Ferner sieht man kleine zackige Gebilde oder unregelmässige Platten (Fig. 17-28 und 37, Taf. VI), ferner geweihartig verzweigte (Fig. 23, Taf. VI) oder bandförmige verflochtene Gebilde. Besonders bemerkt man sie anfangs am Rande der Vakuolen (Fig. 17, Taf. VI), oder an den in Innern derselben befindlichen Faden (Fig. 36 u. 37, Taf.VI).

Auch die in den verdickten $Z$ wischenwanden der vaknolisierten Zellen vorłandenen feineren histologischen Details sind bemerkenswert. Die Zwischenwände werden auseinander getrieben, indem zwischen ihren Fasern sich ăhnliche Fasernetze bilden, wie die in den Vakuolen vorhandenen. Die Faserbildung lasst es nun zu besonders typischen Bildern kommen. So sieht man häufig in der Mitte zwischen den Faserzügen kleine quere Verbindungsfaden, wodurch perlschnurartig aneinandergereihte, kleine, runde oder eckige Vakuolen erzengt werden (Fig. 25, 33, 
$41,43,44,45$, Taf. VI). Weiter können in den dreieckigen Zwickeln Figuren zum Vorschein kommen, wo zwischen quergefaserten Schenkeln von der Spitze zur Basis Vakuolen von zunehmender Grösse enthalten sind (Fig. 41 u. 43, Taf. VI). Ferner können auch Massen von albuminoider Substanz zwischen den Fasern sich ausbilden, welche auf dem Durchschnitt schmale längliche (Fig. 42, Taf. VI) oder konzentrisch ineinander laufende Linien (Fig. 45, Taf. VI) darstellen. Endlich können Fasernetze von verschiedener Dichtigkeit auftreten und sich mit den früher bei den Vakuolen beschriebenen Bildungen in vielgestaltiger Weise komplizieren. In gleicher Art wie an den Vakuolen tritt auch hier die chondromukoide Metamorphose auf. Dieselbe nimmt weiterhin mehr und melır $\mathrm{zu}$ und ergreift schliesslich in mehr allmallichem oder schnellerem Fortgang den gesamten Inhalt der Vakuolen und ihrer Scheidewinde. Zuletzt kommt es zur Bildung hmer oder weniger lomogener, chondromukoid metamorphosierter Massen, in welchen man oft noch Züge früherer Zellwände und Zellmembranen nachweisen kann (Fig. 9, Taf. IV)

Auffallig ist es, dass bei allen diesen Veranderungen der vakuolisierten Chordazellen die nachst benachbarten oft ganz normal erscheinen und dass die Übergänge von den einen $z u$ den anderen vielfach ganz anvermittelt sich darstellen, oft ohne, dass eine Ansammlung von embryonalen Zellen bemerkbar wäre, insbesondere ohne irgendwelche von diesen ausgehende Gewebsbildung (Fig. 9, Taf. IT; Fig. 5, Taf. V). Auch scheint das Endoplasma der vakuolisierten Chordazellen, obwohl lebenskräftig, nur selten sich zu vermehren (Fig. 31, Taf. VI).

Die Bildung des Chordaknorpels hat im allgemeinen die Tendenz von der Peripherie gegen das Zentrum vorzuschreiten; es unterliegt aber nach meinen Untersuchungen keinem Zweifel, dass sich auch unabhängig von den peripheren Partien mehr zentral gelegene bilden können, welche von Anfang an ähnlich wie die vorher geschilderten lediglich durch eine chondromukoide Metaplasie vakuolisierter Chordazellen entstanden sind (Fig. 9, Taf. IV; Fig. 7, Taf. V).

An dieser Stelle möchte ich hervorheben, was auch von der Mehrzahl der neueren Autoren nachgewiesen wurde, dass ich die Elastica externa für gewöhnlich intakt zwischen dem 
Chordaknorpel und dem skelettoblastischen Gewebe verlaufen gefunden habe. In seltenen Fällen konnte ich woll kleine winzigeUnterbrechungen, wie $\mathrm{L}$ w off sie beschrieben hat, an ihr konstatieren, aber gerade an diesen Stellen war nichts zu bemerken, was an eine Einwanderung von Zellen des perichordalen Gewebes bätte denken lassen können. Ich konnte sogar halufig eine partielle Wucherung des intervertebralen Knorpels oder des. jenigen des oberen Bogens mit starker Hervorwölbung desselben gegen die Chorda hin konstatieren (Fig. 6, Taf. IV), olne dass die Elastica, abgesehen von einer geringen Verdünnung, irgend eine Läsion ihres Gewebes gezeigt hätte. Ihre Kontinuität war nirgends unterbrochen.

Was nun den auf die beschriebene Weise gebildeten, den grössten Teil des vertebralen Chordaquerschnitts einnehmenden Knorpel betrifft, so lässt er längere Zeit einen Teil komprimierten Chordagewebes in seiner Mitte frei, den sogenannten Chordistrang. Ich habe gefunden, dass die Bildung des Chordastranges nicht allein durch eine Kompression des von der Peripherie her andrängenden Knorpels entsteht, da die Anfange desselben sich schon zu einer früheren Zeit bilden, wo von einer nennenswerten Kompression des Chordagewebes noch keine Rede sein kann. Ich möchte vielmehr einen Teil seiner Bildung schon auf Rechnung der Veranderungen setzen, wie sie bereits anfings und besonders im zentralen Teil der Chorda als Früherscheinuugen der Chondrogenese in Form von Verdickung und Trübung der Scheidewände der Chordazellen auftreten. Weiter habe ich anch gefunden, dass der Chordastrang ein eigentümliches farberisches Verhalten zeigt, da er sich durch eine ausgesprochene Acid ophilie auszeichnet. Während sonst die Scheidewände und Membranen des Chordagewebes mit sauren Farben nur eine schwache Färbung annehmen, fürbt sich der Chordastrang und zwar nur im Bereich des Knorpels oder doch nur wenig darüber hinaus intensir mit sauren Farben: so mit Eosin leuchtend rot (Fig. 7, 10, Taf. V), mit Kresylviolett intensiv blau, mit Lichtgrün smaragdgrün, mit Pikrinsăure intensiv gelb. Diese farberische Eigenschaft ist um so auffallender, als sonst solche dem Knorpel dicht anliegenden Faserzüge, wie Studnička beschrieben hat, durch das Chondromukoid des benachbarten Knorpels eine ihm ähnliche basische Färbung annehmen. Der Grund der stärkeren Acidophilie des 
Chordastranges ist auch nicht etwa daran gelegen, dass hier der fürberische Effekt mehrerer zusammenliegender Chordafaserzüge sich zu einer starkeren Gesamtwirkung summiert, denn auch die meh" vereinzelt im zentralen Gebiet des Chordakorpels liegenden Faserziige färben sich meist in gleich intensiver Weise mit sauren Farben wie der Chordastrang. Es scheint mir hier ein Verhalten vorzuliegen wie beim Vorknorpel, bei welchem die Scheidewände sich auch mit sauren Farben farben, ehe sie später eventuell die chondromukoide Farbung annehmen.

Der fertige Chordaknorpel zeigt in seinen frühesten Entwicklungsstadien noch zahlreiche Nerkmale, welche an die Art und Weise seiner Entstehung erinnern (Fig. 10, Taf. V). Was die \%ellen betrift, welche teils von den Chordaepithelzellen, teils von den Endoplasmazellen der vakuolisierten Chordazellen sich herleiten, so kann ihre Zahl, Dichtigkeit der Lagerung, ihre Grösse, Form und Beschaffenheit, sowie ihre Verbindung mit der Kapsel und die Ausbildung der letzteren je nach dem Alter des Knorpels eine sehr mannigfaltige sein. Im Anfang sind die Zellen hïufig noch zahlreich, dichter gelagert, mit chromatinreichen Kernen versehen und reich an Protoplasma (besonders bei Salamandra maculata) und zeigen noch vielfach innigen Zusammenhang des Protoplasmas mit der Kapsel, sodass bei der Fixation zablreiche kurze, feinste Ausläufer, welche mit Vorliebe die Knorpelfürbung annehmen, zwischen Protoplasma und Kapsel zu bemerkell sind. Daneben gibt es aber auch noch Zellen, welche nur wenig perinukleäres Protoplasma haben und lange, meist breitere Protoplasmaauslaufer zur Kapsel, gelegentlich auch in die feinfaserige Grundsubstanz schicken. In letzterem Fall ist die Kapsel auch nur wenig ausgebildet. Ferner gibt es Zellen mit weitmaschigem Protoplasma, welches sich an einzelnen, auf dem Durchschnitt halbmondförmigen Partien der Peripherie verdichtet (Fig. 40, Taf. VI). Dieselben färben sich ahnlich den peripheren zur Kapsel gehenden Protoplasmafortsätzen gern mit Knorpelfarbstoffen. Häufig sieht man grössere oder kleinere Vakuolen entweder leer oder mit dichtem, die Knorpelfärbung annehmendem Fasernetz (Fig. 39, Taf. VI), ferner auch mehr oder weniger intensiv basophil gefärbte Flecken von der Grösse der Knorpelzellen. Sie sind als Knorpelzellen aufzufassen, welche nur noch undeutlich oder schattenhaft an ihre frühere Existenz 
erinnern und im Begriff stehen, sich in Knorpelgrundsubstanz umzuwandeln ("verdammernde Zellen", Sch affer [38]). Auch gibt es Zellen, deren Kerne arm an Chromatin oder in Degeneration sind, welchen das Schicksal der eben geschilderten Zellen bevorsteht. Gelegentlich kommen Vakuolen vor, welche kernloses I'rotoplasma von der Grösse einer Knorpelzelle einschliessen (Fig. 6, 'Taf. V), oder Vakuolen mit ringförmigem I'rotoplasma mit oder olne Kern, ferner Kapseln mit zwei Zellen. Zuweilen sind auch Knorpelzellen mit unregelmassig geformter Kapsel zu beobachten.. Einmal konnte ich eine Zelle konstatieren, welche von Knorpelgrundsubstanz dicht umgeben war, während durch einen schmalen Saum von ihr getrennt sich eine kleine leere Vakuole vorfand. Häufig sind auch Chondrintröpfchen sowohl im Faserwerk der Zellen und am Rande der Vakuolen, als auch in der Grundsubstanz, besonders im Bereiche der verknorpelten l'aserscheide, vorhanden. Dort sind sie gewöhnlich grösser und oft zackig. (Fig. 6, Taf. V).

Die Grundsubstanz ist teils homogen, teils fein faserig und zeigt im jungen Chordaknorpel oft recht unregelmässige basophile Färbung, wodurch häufig eine verwaschene Zeichnung mit abwechselnd heller und dunkler gefürbten Stellen entsteht. Besonders um die Kapseln herum ist die Grundsubstanz meist. stärker gefärbt und feinfaserig, oft daselbst auch konzentrisch geschichtet mit abwechselnd dunkleren und helleren Schichten (Fig. 10, Taf. V). Von der Faserscheide ist im vorgeschrittenerer Stadium der Knorpel meist nicht mehr abzugrenzen. Das Knorpelgewebe reicht dann bis an die Elastica externa; man kann dann typische Knorpelzellen mit Kapsein nahe an der Elastica externa bemerken. In jedem Falle bleibt aber die Elastica externa deutlich als Grenze bestehen; von einer Einwucherung von Elementen des äusseren Knorpels ist nichts zu bemerken.

In einigen Präparaten fand ich eine durch Alkohol hervorgerufene netzförmige Beschaffenheit der Grundsubstanz. Wenn solche Artefakte auch keine weitere Bedeutung haben, so zeigen sie doch vielleicht, dass, wie auch Flesch dies in ahnlichent Fällen annimmt, hier dichtere Partien der Knorpelgrundsubstanz mit weniger dichten abwechseln. Auch Bütschli konnte durch geeignete Behandlung des hyalinen Rippenknorpels des Kalbes (Einlegung in Alkohol und Austrocknung unter der Luftpumpe) 
ein aus Interzellularsubstanz bestehendes Wabennetz in der Grundsubstanz sichtbar machen, was für gewöhnlich der Beobachtung entging.

Nach vollendetem Larvenleben nimmt der Zellenreichtum des Chordaknorpels ab. Auch färbt sich die Grundsubstanz dann streckenweise mit basophilen Farben weniger gut (metachondrales Stadium). Der Chordastrang (Fig. 10, Taf. V) schwindet im Verlaufe des Verknorpelungsprozesses auch mehr und mehr; der ('hordaknorpel nimmt dann durchweg in seiner Grundsubstanz eine mehr homogene Beschaffenheit an.

Bezüglich der Bildung des Chordaknorpels in den einzehnen Wirbelabschnitten ist bekannt, dass er im Schaldelteil der Chorda beginnt und allmählich nach dem Schwanzende $\mathrm{zu}$ fortschreitet Im Schädelteil sowohl wie in der Schwanzpartie zeigt die Bildung des Chordaknorpels jedoch häufig Unregelmässigkeiten In der Schidelchorda ist die Verknorpelung meist in unregelmässiger Weise über grössere Strecken verbreitet und ist dort oft noch in den Anfängen, wänrend sie in den oberen Rumpfpartien schon weiter fortgeschritten ist. Ebenso kann auch in der Chorda des Schwanzes manchmal eine Wirbelpartie in der Verknorpelung erheblich gegen die benachbarten Partien zurückbleiben.

Am Schlusse dieses Kapitels ist ü ber die Bildung des Chordaknorpels beim Salamander und Triton noch zu sagen, dass sie im wesentlichen gleich verlauft wie beim Axolotl, indessen, wie früher schon gesagt, spielt sich hier der Werdegang des Prozesses weniger deutlich $a b$, da die zelligen Elemente kleiner sind und die Verknorpelung in melir unregelmissiger Weise verlauft Bemerkenswert ist, dass ron Anfang an neben den vertebralen auch die intervertebralen Partien der Chorda Veränderungen zeigen, welche in Form von fädigem, chondromukoidem Gerüstwerk innerbalb der vakuolisierten Zellen der Chorda sich darstellen. Fs besteht hier nicht, wie beim Axolotl, ein ausschliesslich auf die vertebralen Teile, beschranktes Frühstadium der Chondrogenese. Auch ist die Bildung des Chordaknorpels häufig eine mehr insuläre. Man findet dann isolierte zentrale Knorpelinseln neben den peripheren und dies nicht nur in vertebralen, sondern auch in intervertebralen Teilen (Fig. 7, Taf. V). Von den peripheren Partien sind diese oft durch dicke 
Strïnge komprimierten Chordagewebes getrennt. Noch am Ende des Larvenlebens kommt es zu ausgedehnter Verknorpelung nicht nur im vertebralen Teil, sondern auch in dem relativ engen, intervertebralen Teile der Chorda. In letzterem zeigt die Chorda vor der Verknorpelung chordastrangähnliche Verdickung der Zellmembranen und Verkleinerung der Vakuolen. Beim Axolotl kommen intervertebrale Verknorpelungen nur andentungsweise bei alteren Tieren vor. Auf die bei erwachsenen 'Tritonen und Salamandern weiter vor sich gehenden Veränderungen der Chorda durch Markraumbildungen und gelenkartige Bildungen in den Intervertebralteilen gehe ich hier nicht ein.

\section{Regenerationsversuche.}

Wenn ich auch im allgemeinen die Resultate von Barfurth, Fraisse und von $\mathrm{Nusb}$ a $\mathrm{m}$ und Sidoriak bei der Regeneration des Schwanzendes des Axolotls und der Forelle bestittigen konnte, so haben doch meine Resultate einige erwahnenswerte Besondelheiten anfuweisen. Wie bereits $B$ arfurth hervorgehoben hat, macht es in bezug auf die Art und die Schnelligkeit der Regeneration des Schwanzendes einen wesentlichen Unterschied, ob man die Embryonen im $\mathrm{Ei}$ amputiert hat, oder erst später, nachdem sie ausgeschlïpft sind. Ich habe von solchen Embryonen der ersten Kategorie nur den Axolotl untersuclet (Fig. 13, Taf. VI). Hier bildet sich der Chordastab, (Barfurth, nach anderer Bezeichnung Knorpelstab) als welcher sich die regenerierte Chorda späterhin darstellt, vorzugsweise aus den Chordazellen des unteren Chordaendes und zwar sowohl der epithelialen Randzellen, als auch der in ihrer nüchsten Nähe befindlichen vakuolisierten Zellen. Trotz ihrer Differenzierung haben letztere einen noch jugendlichen Charakter und ist ihr Endoplasma sebr geneigt, sich durch Teilung zu vermehren, wobei zahlieiche Kernteilungsfiguren auftreten. Die durch Teilung entstandenen neuen Zellen bei diesen ganz jungen Axolotlembryonen besitzen ein durch Hämatoxylin stark färbbares Protoplasma und zeigen in ihrer Umgebung Züge und Netzwerk von Protoplasmafasern, welche bald die chondromukoide Umwandlung erfahren. Die von Barfurth beschriebene, bei der Regeneration ganz junger Embryonen auftretende Umwandlung neugebildeter Chordazellen in grosse hyaline Zellen, welche später zu Zellen des Chordastabes wurden, ent- 
spricht vielleicht diesen grossen durch Teilung entstandenen Zellen am Übergange des Chordaendes in den Chordastab. Neben der genannten Bildungsweise des Chordastabes konnte ich aber bei diesen ganz jungen Axolotlembryonen auch eine meta plas t is che Umwandlung bereits differenzierter Chordazellen beobachten. Diese tritt häufig etwas proximalwărts vom Chordastab an einer kleineren Zahl von Chordazellen auf, in Form kleiner Netzbildungen oder mehr gröberer, unregelmässig geformter albuminoider Massen im Innern der Valiuolen, in ähnlicher Weise, wie wir solche bei der Bildung des Chordalinorpels beschrieben haben. Auch die chondromukoide Umwandlung oder Ausscheidung erfolgt ähnlich wie dort in umregelmässiger Ausdehnung früher oder später.

Was die Beteiligung von perichordalen Zellen an der Bildung des Chordastabes betrifft, so konnte ich weder bei ganz jungen Embryonen, noch auch bei alteren eine solche mit Sicherheit nachweiseisen.

Jedenfalls ersehen wir ans dem mitgeteilten Befunde, dass auch bei gallz jungen, im Fi amputierten Embryonen vom Axolotl schon in früher Zeit die ersten Anfänge der Chordastabbildung sich bemerkbar machen. Nach Barfurth soll dieselbe hier erst in späterer Zeit erfolgen, nachdem ein Stadium der Bildung von grossen, hyalinen Chordazellen aus dem alten Chordagewebe rorausgegangen war.

Die Regeneration bei alteren Embryonen, von welchen ich $A$ xolotl und Forellen untersuchte, verläuft wesentlich langsamer und in einer von der beschriebenen etwas verschiedenen Weise. Erst einige Tage nach der Amputation des Schwanzes ron Forellen- und Axolotlembryonen findet eine Überhäutung des stark vorgetriebenen Chordaendes durch das Lithel der Epidermis statt, dessen Zellen gleichzeitig eine starke Wucherung und Vermehrung im Bereiche der Chordaendes darbieten. Bei der Forelle kann man ausserdem eine starke Vermehrung der Chordaepithelzellen ziemlich weit proximalwärts innerhalb der Chorda konstatieren. Ich möchte hier hervorheben, dass das Chordaepithel der Forelle überhaupt zu Wucherungs- und Vermebrungsprozessen sehr geneigt ist und auf Reize jeglicher Art sowohl auf aussere als anch auf solche, wie sie durch ein regeres Wachstum besonders am unteren Chordaende bei der Bildung des 
Chordastabes gesetzt werden, stark reagiert. Das alte, stark abgeplattete Chordaepithel wird nun bei der Forelle durch das nachwuchernde neue Epithel oft an manchen Stellen in Form eines flachen Saumes abgehoben und nach innen gedrängt. Dieser Saum nimmt mit Pikrinsäure meist eine gelbliche Färbung an und kann auch später noch zu faserartigen Bildungen atrophieren, welche oft in einem nach dem Chordaende zu konvexen Bogen von einer Seite der Chorda zur anderen verlaufen. Hie und da kommt es zu Wucherungen der Faserscheide, wobei sich nach innen ziehende Fasern und Falten bilden können. Beim Axolotl erfolgt die Vermehrung des Chordaepithels nur in mässigem Grade, aber öfters unter Auftreten schöner Kernteilungsfiguren und beschränkt sich nur auf die năchste Umgebung des unteren Chordaendes. Bei der Regeneration des Schwanzes alterer Embryonen war es mirim Anfange der Entwicklung nicht möglich, metaplastische Prozesse an den vakuolisierten Chordazellen uachzuweisen. Es scheint, dass hier, wo der Umbildungsprozess von Chordagewebe in Knorpelgewebe sich auf eine langere Zeit verteilt, als bei der Regeneration ganz junger Embryonen, die am meisten distalwärts gelegenen und zwar sowohl die protoplasmatischen als die vakuolisierten Chordazellen genügen, um durch Teilung und Vermehrung das Knorpelgewebe des Chordastabes zu bilden. Erst in etwas späterer Zeit, wenn der Chordastab an altere. vakuolisierte Zellen grenzt, sieht man beim Axolotl sowohl, als auch bei Forellen neben der Knorpelbildung vom Chordaepithel auch solche durch metaplastische Prozesse an den vakuolisierten Zellen in Form von Tröpfchen- und Netzbildung Platz greifen. Auch konnte ich in einiger Entfernung vom ausgebildeten Chordastabe junger Axolotl isolierte Knorpelherde in der Chorda finden, welche mit der Faserscheide in Verbindung standen und als unregelmässige Bildungen von Chordaknorpel aufzufassen sind, wie sie in diesem unteren Chordaabschnitte gewöhnlich angetroffen werden.

Bezüglich der normalen Entwicklung des Chordastabes möchte ich noch bemerken, dass auch hier eine Einwanderung perichordaler Zellen nicht stattfindet und metaplastische Prozesse an den vakuolisierten Chordazellen erst im späteren Stadium des Entwicklungsprozesses vorkommen. Der Zeitpunkt der Bildung des Chordastabes ist auch nicht an eine bestimmte 
Lănge des Tieres gebunden. Wenigstens konnte ich bei Tieren von gleicher Länge (etwa $2 \frac{1}{2} \mathrm{~cm}$ bis $3 \mathrm{~cm}$ ) zuweiler einen wohl entwickelten Chordastab nachweisen, zuweilen aber auch nichts von einem solchen, sondern lediglich eine Chorda, deren unteres Ende aus Chordaepithel und wohlgebildeten vakuolisierten Zellen bestehend von einer dünnen Fortsetzung der Faserscheide umgeben war und bis nahe an den Hautsaum des Schwanzes reichte. Worauf diese Verschiedenheit beruht, vermag ich nicht anzugeben, vielleicht sind die bei diesen Tieren öfters vorkommenden kleinen Verletzungen des Schwanzendes von Einfluss hierauf.

Der Chordastab wahst nun aber wie die laufig im Innem seines Gewebes zu findenden Kernteilungsfiguren und 'Tochter'zellen beweisen, auch durch Intussusception. Ich möchte diesem Wachstum besonders später, wo der Teilungs- und Vermehrungsprozess in den Zellen des untersten Chordaendes kein so reger mebr ist, eine grössere Bedeutung beimessen als dem chordalen Wachstum.

\section{Kontinuitatstrennungen der Chorda.}

Bei einer jungen Forelle von ca. $3 \mathrm{~cm}$ Länge wurde eirr Schnitt unterhalb des Afters angelegt, welcher etwas schräg verlaufend, die Chorda vollstandig durchtrennte. Das Schwanzende blieb noch soweit in Verbindung mit dem Rumpf, dass eine Anheilung des getrennten Stückes erfolgen konnte. Vier Wochen mach der Operation zeigte sich folgender Befund (Fig. 12, Taf. T). I) as periphere Chordaende, welches noch durch einerr schmalen, auch makroskopisch sichtbaren Streifen eines zellenreichen Narbengewebes von der übrigen Chorda getrennt nnd etwas dorsalwärts verschoben ist, wird von einer gutgebildeten Scheide umgeben und endigt mit spitzer Form nahe der dorsalen Epidermis. Von einem Chordastab ist noch nichts $\mathrm{zu}$ bemerken. Die Chorda dieses peripheren Stückes besteht grösstenteils aus embryonalen, dicht aneinander liegenden Zellen, welche im Bereiche der zentralen Partien weniger gutgefarrbte Kerne zeigen, als in den peripheren Partien. Auch sind dort die Kerne bäutig durch breitere Protoplasmapartien ohne deutliche Zeligrenzen voneinander getrennt. Einzelne abgeflachte Zellen - alte Chordaepithelzellen -, welche sich mit Pikrinsăure gelb färben, sind hie und da eingestreut. Ausser diesen kleinen Zellen und breiteren Protoplasmamassen findet man nur in der Mitte grössere und 
kleinere Vakuolen. Erstere sind manchmal von einem Kranz schmaler, oft etwas länglicher Zellen umgeben. Interessant ist das Verhalten des zentralen Chordaendes. Dort, wo die alte Chordascheide aufhört, wölbt sich ein breiter Chordastumpf ventralwärts mit etwas zugespitztem Ende bis dicht an die ventrale Epidermis vor. Die Vakuolen dieser Chordazellen sind stark elweitert, meist obne Endoplasma und häufig mit einer feinkrümeligen Masse angefüllt. Zwischen diesen vakuolenhaltigen Zellen liegen aber auch noch protoplasmareiche Zellmassen, deren Grenzen nicht immer scharf sind, und deren Kerne meist peripherwarts besser gefärbt sind, als in den immeren Partien des Stumpfes. Einzelne dieser Zellen sind schmal und länglich und besitzen ein mit Himatoxylin sich intensiv blau fïrbendes Protoplasma und können mit den von Strasser beschriebenen dunkeln, prochondralen Elementen verglichen werden. Es ist mir wahrscheinlich, dass der Druck der sich ausdehnenden Yakuolen das Zustandekommen derselben veranlasst hat und dass in einem späteren Stadium des Prozesses es hier zu einer Bildung ron echtem Knorpelgewebe gekommen wäre, in ähnlicher Weise, wie Nusbanm und Sidoriak dies bei der Regeneration des Scliwanzendes der Forelle besclurieben haben. Die Chordascheide hat sich an der ventralen Seite des Stumpfes regeneriert. Dort ïberzieht sie im Anschluss an die alte Scheide in Form einer dümnen, homogenen Haut den sich vorwölbenden Chordastumpf. Auch sieht man dort einen aus schmalen Zellen gebildeten, nach innen verschobenen Saum alter Chordaepithelzellen, welche sich mit Pikrinsiure gelb gefürbt haben. Die Chorda zeigt vom ursprünglichen, zentralen Schnittrande aus eine proximalwarts weitreichende Wucherung des Chordaepithels, welche sich bis in die zentralen Chordapartien erstreckt und hier die Vakuolen vielfach komprimiert und verdrängt lat.

IV. Transplantationen.

Es wurden mehrfach Versuche in der in einem früheren Kapitel năher erörterten Weise vorgenommen und zwar zum Zwecke, den Effekt der durch die Transplantation herbeigeführten Entspamnung des Chordagewebes zn studieren, insbesondere um zu erforschen, ob das Chordagewebe sich dabei etwa in Knorpelgewebe verwandeln würde. In letzterer Hinsicht ergab sich 
zwar ein negatives Resultat; immerhin war es aber interessant, zu ersehen, dass die ausgebildete Chorda dabei sich wieder dem embryonalen Typus näherte, $d . h$. die alte Chorda wieder jung wurde, und zwischen den Membranen breite Protoplasmaschichten gebildet wurden, wobei es $\mathrm{zu}$ einer allmählichen Verkleinerung und schliesslich zum Schwunde der Chordavakuolen kam. In den ersten Wochen nach del 'Transplantation finden sich keine Veränderungen vor. Erst nach Ablauf von drei bis vier Wochen können sich bemerkenswerte Veränderungen ergeben, falls die Einheilung glatt ohne stärkere Reaktion in der Nachbarschaft erfolgt. Es kommt aber auch vor, dass selbst nach dieser Zeit das Transplantat noch wenig verïndert ist. Es hängt dies offenbar von anderen Bedingungen noch ab, wie Grösse nnd Form des Transplantats, Ernalhrungsbedingungen desselben, Zirkulationsverhältnisse, Beschaffenheit des Nihrbodens etc.

Einige der wichtigeren Befunde lasse ich hier unter. Verweisung auf die Abbildungen folgen. Fig. 13, Taf. VI und Fig. 14. Taf. IV stellen einen Fall vor, wo das vier Wochen alte Transplantat eines $3 \mathrm{~cm}$ langen Axolotls an einer schmalen Stelle nit dem intermuskulären Gewebe zur Verheilung gelangt war; während ringsherum sich ein ziemlich weiter Lymphraum gebildet hatte, in dessen Flüssigkeit einige Lymph- und Endothelzellen suspendiert waren. Das Gewebe der Chorda zeigte teilweise noch die valkuolisierten Chordazellen mit dazwischenliegenden breiten Protoplasmazügen, teilsweise fand sich aber ein aus grossen Riesenzellen bestehendes Gewebe, welches nach der Peripherie hin in ein feinfaseriges, an Spindelzellen reiches Bindegewebe überging. Die durch das zwischengelagerte Protoplasma stark verkleinerten Vakuolen waren meist kernlos; in das Protoplasma waren bereits einige Granulationszellen eingedrungen. Ein anderes Präparat (Fig. 15, Taf. IV), ebenfalls vier Wochen alt, $3 \mathrm{~cm}$ lang, war in seiner ganzen Limgebung zur Einheilung gelangt. Hier war eine deutliche, wenn auch in ihrer Struktur veränderte Elastika vorhanden. Von der Faserscheide war nichts mehr zu sehen. Im Chordagewebe fanden sich noch einzelne grosse und kleine Vakuolen, welche mit einem protoplasmatischen, feinwabenartigen Netzwerk erfüllt waren. Die Vakuolen zeigten keine Kerne im Innern. Ihre Wand war an den grösseren noch deutlich vorhanden und gut von der 
Umgebung abgegrenzt, an den kleineren begann sie stellenweise sich zu verdünnen. Die Vakuolen waren weit voneinander getrennt durch breite Züge eines aus protoplasmareichen Zellen mit grossen hellen Kernen bestehenden Gewebes. Einige Kerne zeigten schöne Kernteilungstiguren. An der âsseren Umrandung der Vakuolen lagen einige schmale chromatinreiche Kerne. Ein drittes Prïparat (Fig. 16, Taf. IY) möchte ich der Vollständigkeit wegen noch erwainen, welches, wenn auch für die vorliegenden Untersuchungen von keinem Belang, dennoch nicht olme Interesse sein dürfte. Das Transplantat stammte von einem etwas grösseren, etwa $4 \mathrm{~cm}$ langen Axolotl. Die Transplantation hatte hier zu einer stïrkeren Haemorrhagie und entzündlichen Reaktion in der Umgebung geführt. Das Transplantat lag innerhalb eines uiber die Obertlache der Haut hervorragenden Granulationsknopfes. Man sieht hier auf dem Querschnitt die ron zahlreichen Querspalten zerklüftete, junge Faserknochenschicht des Wirbels, in der Cimgebung zahlreiche rote und einzelne weisse Blutkörperchen. Im Inner'n findet sich eine breite, stark eingefaltete Yembran, welche wohl als die geefuollene, ursprüngliche Faserscheide der Chorda zu betrachten ist. Im Innern sieht man ferner noch Riesenzellen, grössere spindel- und sternförmige Zellen mit grossen hellen Kernen, sowie auch einzelne grosse Zellen mit wahrscheinlich durch Blutfarbstoff briunlich gefärbtem Protoplasma, weiter noch mehrfache rote Blutkörperchen und einen Gefässquerschnitt.

Bei einer längeren Dauer des Prozesses würde es wohl in allen diesen Fälen zu einer allmählichen Resorption der Chorda und zur Bildung von Bindegewebe an illrer Stelle gekommen sein.

\section{Besprechung der eigenen und der fremden Befunde.}

Um die geschilderten zurVerknorpelung der Chorda führenden Prozesse zu erklären, ist es erforderlich, die anatomischen Befunde in Einklang zu bringen mit den entsprechenden jeweiligen $f u n k t i o n e l l e n A n s p r i i c h e n$, welche an die Chorda in den einzelnen Stadien des Larvenlebens gestellt werden.

Yon wesentlicher Bedeutung ist hierbei, wie auch Gegen baur schon hervorhob, das Auftreten der Verknöcherung im perichondralen Gewebe des Wirbelkörpers, welche zur Bildung einer knöchernen Wandung um die Chorda führt. Solange eine solche 
fehlt, stellt die Chorda ein Rohr dar, dessen Wandung wir uns im wesentlichen durch die elastische Scheide, dessen Inhalt durch die blasigen, mit Flüssigkeit gefüllten Chordazellen gebildet, vorstellen können. In letzterem herrscht nun nach 0 . Hertwig, dessen in seinem Lehrbuch "Die Zelle und die Gewebe" enthaltenen Anschauungen wir hier wiedergeben, infolge osmotisch wirksamer Substanzen der Zellflüssigkeit ein betrüchtlicher Druck, durch welchen die festen Membranen der Zellen prall gespannt werden. Die Druckkräte dieser zahlreichen kleinen turgeszenten Chordazellen summieren sich nun zu einem betrïchtlichen Gesamtdruck, durch welchen die gemeinsame Scheide, besonders die Elastica externa, in Spannung erhalten wird. Wie Ussow berichtet, hat diese Druckspannung eine solche Grösse, dass bei Befreiung aus dem Tierkörper die Chorda viermal länger wird und auch an Volumen bedeutend zunimmt. Auch ist es eine bekannte Tatsache, dass bei Amputation des Schwanzes infolge des Innendruckes ein beträchtliches Stück Chorda wurstförmig hervorgetrieben wird. (Siehe auch Fig. 12, Taf. V, wo das zentrale Stumpfende der Chorda nach der Kontinuitätstrennung stark hervorgetrieben wurde.) Wie nun bei einem Gummischlauch durch pralle Füllung mit Wasser die Druckfestigkeit bedeutend zunimmt, so besitzt auch die, wie vorhin erörtert, in ihrer Elastica prall gespannte Chorda eine ansehnliche mit Elastizitit gepaarte Festigkeit und kann so erst ihre Funktion, dem Tierkörper gleich einem biegsamen Stabe als Stütze zu dienen, ausüben. Es ist nun ein allgemeines Gesetz, dass alle Momente, welche die Funktion eines Organes ändern, mebr oder weniger auch eine Inderung im histologischen Bau desselben herbeiführen. Als ein solches Moment ist nun das A uftreten der knöchernen Umhüllung der Chorda anzusehen. Hierdurch wird die Funktion der Chorda im Sinne einer Entlastung geilndert. Die Druckspannung im Innern der Chorda ist jetzt unnötig geworden und wird nach und nach eine Abnahme erfahren, indem mehr und mehr die knöcherne Hülle die Stützfunktion übernimmt. Die Veränderungen des Chordagewebes infolge der Entlastung werden nun an denjenigen Stellen der Chorda sich am meisten ausprägen, wo zuerst und am ausgiebigsten die umgebende Knochenschicht angelegt wird. Dies ist die Mitte des Wirbelkörpers. Hier sehen wir den Chordaknorpel zuerst 
und in ausgezeichneter Weite gebildet werden, während der Intervertebralteil meist frei bleibt oder erst später zur Verknorpelung gelangt. Es könnte aber bei der Anlage des Chordaknorpels in der Mitte des Wirbels auch der Umstand eine Rolle spielen, dass hier meist der Querschnitt des Wirbelkörpers ein kleinerer ist und deshalb hier der Knochen noch einer besonderen Festigkeit in seinem Innern bedarf.

Schwierig ist nun die Frage zu beantworten: welche feineren Verhältnisse werden durch die Funktionsänderung der Chorda am meisten getroffen und in welcher Weise fübren sie zur Knorpelbildung? In erster Linie liommt nun die $\mathrm{Ab}$ nahme der Druckspannung im Innern der Chorda in Betracht, welche, wie wir gesehen haben, nuumehr entbehrlich geworden ist. Es ist begreitlich, dass mit dem Nachlassen des Flüssigkeitsdruckes in den einzelnen Zellvakuolen auch das Exoplasma oder die Zellmembranen, vielleicht auch die zwischen den Membranen sich befindende Kitt- oder Interzeliularsubstanz, wie wir solche wohl annehmen müssen, die Möglichkeit laben, ein stärkeres Wachstum zu betätigen und hierdurch dem Charaliter des Knorpelgewebes sich nähern. In dieser Beziehung haben auch die von mir in der Absicht vorgenommenen Transplantationsversuche, die Wirkung der Entlastung und Entspannung der Chorda zu untersuchen, das bemerkenswerte Resultat ergeben, dass nach drei bis vier Wochen sich an Stelle der durch die Membranen der vakuolisierten Chordazellen gebildeten Scheidewände breite protoplasmatische Züge bilden, während die Vakuolen sich mebr und mehr verkleinerten, bis schliesslich ein Gewebe entstand, welches allerdings kein Knorpelgewebe war, aber in Übereinstimmung mit den von Ribbert an den verschiedensten transplantierten Geweben gemachten Erfahrungen dem embryonalen Chordagewebe sehr ahnlich war. Aus diesen Transplantationsergebnissen ist aber auch die wichtige Tatsache zu entnehmen, dass die vakuolisierten Zellen trotz ihrer weitgehenden Differenzierung dennoch imstande sind, anderweitige Umwandlungen zu erfabren. A us den membranösen Exoplasmen sind breite protoplasmatische $\mathrm{Z} u ̈ g e$ entstanden. Hierdurch ist nun auch die Ausicht derjenigen Autoren widerlegt, welche, wie Goette, die ausgebildete, vakuolisierte Chorda für ein seniles, keiner 
weiteren Entwicklung mehr fähiges Gewebe halten. Nach Goet te soll die Vitalität der vakuolisierten Chorda besonders auch dadurch herabgesetzt sein, dass entwicklungsgeschichtlich die Vakuolen nicht je einer Zelle, sondern 'Teilen einer Zelle entsprechen, indem ursprünglich nicht eine, sondern mehrere Vakuolen in einer Zelle sich ausbilden und somit nur eine kleinere Anzahl der späteren Vakuolen kernhaltiges Protoplasma enthielten. Letzteres widerspricht auch den Lntersuchungsergebnissen Fields, welcher - bei Urodelen wenigstens - nur eine Vakuole in jeder embryonalen Chordazelle nachweisen konnte. Zuzugeben ist allerdings, dass bei alteren Tieren atrophische Zustände am Protoplasma und den Kernen der vakuolisierten Chordazellen auftreten kömmen, bei jüngeren Urodelen ist dies jedoch nicht der Fall.

Die Knorpelbildung in der Chorda wïrde nun also mit einer Herabsetzung des Gewebedruckes einhergehen, im Gegensatz $2 u$ dem von Strasser an der Extremitatenanlage von Tritonenlarven beobachteten Modus. Hier spielte eine durch den Wachtumsdruck des jungen Gewebes hervorgerufene Kompression und Einklemmung von Protoplasmamassen eine Rolle und gab zur Entstehung der sogenannten dunklen, sich später in Knorpelelemente umwandelnden Körper Veranlassung. Delartige dunkle Gebilde sind bei der Verknorpelung der Chor'da nicht oder nur selten zu beobachten. Ich fand sie am zentralen Stumpfende einer künstlich getrennten Chorda (Fig. 12, Taf. V), wo die stark ausgedehnten Vakuolen der Chorda die in ihrer Umgebung befindlichen Zellen zu solchen platten dunklen Elementen umgewandelt hatten. $\mathrm{Nusbaum}$ und Sidoriak sahen ebensolche bei der Regeneration des Schwanzendes des Forellenembryos, V. Schmidt, J. Barfurtb und Fraisse im Endteil des Chordastabes.

Ob nun stets der hydrostatische Druck in der Chorda im Verbältnis zur Ausbildung der äusseren Knochenschicht abnimmt, ist schwer zu sagen. Manche Verhältnisse, wie die Entstehung der grossen Vakuolen im Innern der Hechtchorda in der Umgebung des Chordastranges, sprechen dafür, dass im frühen Stadium der Knochenbildung, solange es sich noch um jungen Faserknochen handelt, welcher trotz seiner Rigidităt noch eine bedeutende Elastizität besitzt, vielleicht sogar eine Steigerung

Archiv f. mikrosk. Anat. Bd. 73. 
des Druckes der Abnahme desselben vorausgeht. Die Bildung der Vakuolen könnte aber auch auf andere Weise erfolgell und müsste noch an jungen Hechten untersucht werden, wozu mix leider das betreffende Material fehlte.

Ausser der Abnahme der Druckspannung sind nun aber auch noch vielleicht andere Momente bei der Funktionsänderung der Chorda für die Knorpelbildung in Betracht zu ziehen. Einmal die Änderung der Zirkulationsververhältnisse und die hierdurch bedingte Ïnderung der Ernährung des Gewebes, sowie ferner eine Verkleinerung des Querschnittes der Chorda. Letzteres kann, wie mir scheint, zuweilen durch die äussere Knochenanlagerung herbeigeführt werden, dann aber auch in der Natur der Entwicklung liegen, wie z. B. beim Salamander und Triton, bei denen die Intervertebralpartien der Chorda schon frühzeitig sich beträchtlich verengern und deshalb auch zur Bildung von Knorpel neigen.

Dass die Änderung der Zirkulationsverhaltnisse von Bedeutung für die Knorpelbildung ist und in der Richtung erfolgt, dass die Intervertebralabschnitte eine bessere Ernahrung erfahren, als die Vertebralteile, ist wahrscheinlich; ich möchte aber nicht so weit gehen wie Ussow, welcher die bei Knochenfischen in der Chorda sich ausbildenden grossen Vakuolen als entstanden durch einen Degenerationsprozess infolge mangelhafter Ernährung durch die veranderte Zirkulation auffasst, im Gegensatz zu den durch bessere Ernährung entstehenden mächtigen Intervertebralsepten.

Welches nun auch die feineren, zur Bildung des Chordaknorpels führenden ursächlichen Verhältnisse sein mögen, so ist doch die Tatsache nicht von der Hand zu weisen, dass zeitlich die erste Entwicklung des Chordaknorpels, wie sie sich in der Ausbildung des chondromukoiden Fasergerüstes und in der Verdicking der Scheidewände der blasigen Zellen des Vertebralteils der Axolotlchorda darstellt, mit der ersten Anlage des perichordalen Knochens zusammenfallt und beide deshalb wohl in ursächlicher Beziehung zueinander stehen müssen.

Wie nun die anatomischen Befunde ergeben haben, ist die Knorpelbildung in der Chorda als ein im wesentlichen metaplastischer Prozess zu betrachten, welcher abgesehen von den peripheren Partien ohne Beteiligung von embryonalen oder 
chondroblastischen Zellen (Schaffer) verlauft. Bekanntlich haben diejenigen Autoren, welche eine Entstehung von Knorpel aus der C'horda, einem seiner Herkunft aus dem Entoderm nach epithelialen Ciebilde, für ausgeschlossen hielten, ihre Zuthucht zu solchen indifferenten Zellen genommen. Man hat alsdann angenommen, dass entweder von aussen, vom perichondralen Gewebe, herkommende Zellen durch die Elastica in die Chorda einwanderten ( $\%$ ykoff und studnička) oder man hat, wie jetzt die Mehrzahl der Autoren. die Zellen des Chordaepithels als embryonale, indifterente Bildungszellen angeschen, welche einmal imstande sind. die spezifischen Chordazellen zu liefern, zweitens aber auch dem Stützgewebe angehörige Zellen, wie Linorpelzellen, zu bilden. In jedem Falle aber hat man es für unmöglich gelialten, dass die beleits mehr differenzierten valiuolisierten Chordazellen sich in Kinorpelzellen umwandeln könnten. Klaatsch war der einzige, welcher die Ansicht vertrat, dass möglicherweise neben den Chordaepithelzellen auch die noch wenig vakuolisierten Chordazellen an der linorpelhildung teilnehmen könnten. Wie unsere Lintelsuchingen ergeben haben, ist eine Einwanderung von chondroblistischen Zellen durch die Elastica externa in die Chorda nirgends nachzuveisen. Die früheren derartigen Peobachtungen, besonders von $Z$ ykoff, sind teils auf mangelhafte Technik, teils auch auf 'Täuschungen durch die Schnittrichtung oder wie bei der Schädelchorda auf eine nachträgliche, durch Verdünnung und Einschmelzung del Elastica externa infolge des Wachstumsdruckes hervorgerufene Verbindung des Chordaknorpels mit dem Occipitalknorpel zurückzntühlen.

Es bleibt deshalb übrig, die lediglich auf die Chordazellen zu heziehende Knorpelbildung zu besprechen und wollen wir zmächst die Vorgänge an den Chordaepithelzellen betrachtell. Auch nach unseren Untersuchungen unterliegt es keinem $Z$ weitel, dass dem Chordaepituel ein hervorragender Anteil an der bildung des Chordaknorpels zulkommt und verdanken besonders die peripheren Partien desselben seine Entstehung dem Chordaepithel. Es kann als Einleitung des peripheren Verknorpelungsprozesses eine Teilung und Vermehrung der Chordaepithelzellen stattfinden: immerhin möchte ich aber dem Chordaepithel nicbt die aktive Rolle zuschreiben, wie es diejenigen Autoren tun, welche die Bildung des Chordaknorpels lediglich auf die Tatigkeit 
des Chordaepithels zurückführen. Die Teilung und Vermehrung des Chordaepithels ist besonders im Anfange des Prozesses selır oft noch gar nicht vorhanden, $z u$ einer Zeit, wo, wie oben beschrieben, gerade Veränderungen an den vakuolisierten Chordazellen und besonders an den zentralen in Form von Verdickungen der Zellenmembran und Bildung faserig netzförmigen Gerüstes innerhalb der Vakuolen sich bemerkbar machen. Das Chordaepithel reagiert vermöge seiner innigen Beziehung zur Faserscheide, mit welcher es gewissermaßen einen einheitlichen Apparat darstellt, zunächst nur durch eine stärkere Wocherung einzelner Zellen, welche sich in der Weise kundgibt, dass das Protoplasma in der Umgebung des Kernes und zwar besonder's dort, wo es: mit der Faserscheide zusammenhüngt, in Form netzförmigel Bildungen zunimmt, wodurch die Kerne melı nach immen rücken. und die Faserscheide anscheinend verbreitert wird. In der hege! erst später, wenn auf diese Weise lileine umschriebene Wülste oder Halbinseln sich gebildet haben, liommt es zur Vermehrung und Auswanderung von Chordaepithelzellen in der Lmgebung. wie ich glaube durch den Reiz, welchen die mehr kompakte Masse der Wülste auf die benachbarten Zellen ausübt. Es ist bekannt, dass die mehr jüngeren Zellen des Chordaepithels zu Teilungs- und Vermehrungsprozessen mehr geneigt sind, als die mehrdifferenzierten vakuolisierten Zellen. Meistens werden wohl diese neugebildeten Zellen in die neu sich bildende linorpelgrundsubstanz eingeschlossen, indessen will ich nicht bestreiten, dass sie in manchen Fällen, wie besonders bei Triton und Salamander, auch wohl imstande sind, weiteres Knorpelgewebe aus sich herauszubilden. Beim Axolotl konnte ich solches nicht konstatieren, dagegen habe ich inmitten der Schädelchorda eines $4 \mathrm{~cm}$ langen Forellenembryos eine Anhäufung von embryonalen Zellen gefunden, zwischen welchen sich eine chondromukoide Zwischensubstanz ausgeschieden hatte. Jedenfalls geht die vom Chordaepithel gebildete Knorpelsubstanz nicht über die peripheren Partien der Chorda, d, h, über das Bereich der noch wenig vakuolisierten Chordazellen hinaus. Ich bemerke noch, dass wenn lediglich das Chordaepithel als Knorpelbildner anzusehen wäre, sich wohl durch Kompression der zahlreichen vakuolisierten Zellen und Membranen ein weit mächtigerer Chordastrang bilden müsste, als er für gewöhnlich erscheint. 
Was nun die vakuolisierten Chordazellen betrift, so konnten wir hier, wie schon früher gesagt, keine oder doch böchst selten Teilungs- und Vermehrungsprozesse konstatieren. Es ist auch, wie früher schon hervorgehoben, sehr auffallig, wie hïufig man in der Umgebung der in Verknorpelung begriffenen eigentlichen Chordazellen eine Ansamminug von embryonalen Zellen oder auch sonstige Übergänge zu Knorpelgewebe vermisst. Oft geht das nicht veründerte Chordagewebe ganz piötziich in die schon erheblich umgewandelten Chordapartien über. Dies ist eben nur dadurch erklärlich, dass die Verknorpelung der vakuolisierten Zellen, soweit dieselben nicht durch Schrumpfung oder Kompression im Chordastrang untergehen, durch einen metaplastischen Vorgang erfolgt, und damit kommen wir auf die chondromukoide Metamorphose zu sprechen. Es wirft sich mis besonders die Frage auf, wenn wir die mamnigfaltigen anatomischen Bilder, welche die im Verknorpelungsmozesse begriffienen, blasigen Chordazellen zeigen, betraciten: Wie kommt es zu der Bildung der 'Trijpichen, welche oft zu solchen bizarren l'iguren konthieren und zur Bildung der Netze? Hierauf geben uns nun meines Erachtens die physikalischexperimentellen Ergebnisse von Bütschli und $\mathrm{Hardy}$ eine gute Erklärung. Die kolloiden Stoffe, zu denen auch das Plasmit gelört, treten bekanntlich in zwei physikalischen Zuständen: als leichttlüssige Lüsungen (Solen) und als gelatinierte Substanzen (Gelen) anf. Erstere stellen mehr oder weniger feine Suspensionen dar, williend die gelitinierten Substanzen oder Gele nach B ï tschli eine besondere heterogene Struktur besitzen und aus ersteren durch einen eigentümlichen Entmischungsvorgang ohne Wasserverlust durch innere Umgestaltung hervorgehen. Diese Gelatinierung, welche in der sogenannten Phasenbildung, der Wasserphase und der Gelatinphase zum Ausdruck kommt, wird nach B ïtschli hervorgerufen durch den Einfluss, welchen eine dritte Substanz auf eine Lösung ausübt. Hardy fand, dass je nach der Konzentration, je nach der 'Temperatur und dem Lösungsmedium des Colloids die Gelatinierung unter Bildung von Waben oder feinster Tröpfchen vor sich geht und dass diese Tröpfchen, welche aus der Gelatinphase bestehen, allmählich erstarren und wenn sie sich berühren, zusammenkleben und Netze bilden. Dieses experimentelle Ergebnis erklärt sehr gut die von 
uns an den vakuolisierten Zellen gefundenen histologischerr Details, insbesondere die in den Vakuolen der Chordazellen zur Ausscheidung kommenden Tröpfchen und Netze. Wir kömmen den Vakuoleninhalt der blasigen Chordazellen als eine leicht Hüssige kolloide Lösung betrachten. Unter dem Einflusse der Tatigkeit des eigentlichen Zellprotoplasmas (Endoplasma) kommt es zur Ausscheidung der chondromukoiden Substanz, einer mehr konzentrierten kolloiden Substanz in die Umgebung des Encloplasmas und besonders in die Vaknolenfluissigkeit. Durch das Zusammentreffen dieser Substanzen erfolgt nun eine Entmischung des fiüssigen Vakuoleninhalts, eine sogenannte Gelatinierung oder Gerinnung, welche mit einer inneren Umgestaltung des Vakuoleninhalts verbunden ist, wobei es zunächst zur Bildung ron 'Tröpfchen kommt. Dieselben sitzen zunächst an den festeren Partien des Endoplasmas oder der Vakuolenwande und verbreiten sich von dort, Fäden und Netze bildend, durch den Hohlraum der Vakuolen. Es muss angenommen werden, dass die 'Tröpf'chen in vielen fallen wohl zuerst in form einer nicht färbbarer albuminoiden Substanz ausgeschieden werden und erst später durch weitere chemische Differenzierung sich in Chondromulioid umwandeln, denn die aus den Tröptchen hervorgehenden Fider und Netze nehmen oft längere Zeit die Firloung für (hondromukoid nicht an. Statt der Tröpfchen und Setze können in anderen Fällen sich auch Waben und Membranen bildell. In gleicher Weise wie in den Vakuolen der Chordazellen bilden sich die 'Tröpfchen und Netze auch in den Spalträımen der verdicktent membranösen Scheidewände der Chordazellen, sowie in Ind aur den Maschen der protoplasmatischen Netze der (hordaepithelzellen und der Faserscheide. Die Tröpfchen können nicht nur zu Fäden sich aneinanderreihen, sondern auch zu grösseren Tropfen oder auch zu verschiedenen zackigen oder tropfsteinartigen Gebilden kontluieren. Vielleicht können sich auch voll Anfangan etwas grössere Tropfen bilden. In jedem Falle möchte ich. die grundlegenden, histologischen Formen der chondromukoiden Metamorphose in der Bildung der Tröpfchen und Netze erblicken, durch deren Konfluenz und Verdichtung grössere Gewebspartien der Verknorpelung verfallen oder, ähnlich wie die kollagenen Fasern, derart von diesen Bildungen umgeben oder eingehüllt werden, dass ihre ursprüng- 
liche Struktur unsichtbar wird (Chondromukoide Maskierung; Hansen).

Die in dieser Weise durch die chondromuloide Metamorphose des Inhalts der Vakuolen und der Spalträume der Scheidewände entstandenen Gebilde sind nun nicht als Protoplasma, sondern als den Interzellularsubstanzen, d. h. etwa dem Fibrin oder Mucin gleichwertige Gebilde zu betrachten.

Nachdem wir der Ansicht Ausdruck gegeben haben, dass an der Bildung des Chordaknorpels beide Arten von Chordazellen, sowohl die epithelioformen, als auch die vakuolisierten in gleichem Maße beteiligt sind, sodass der ganze Prozess der linorpelbildung im wesentlichen nur als eine strukturelle und chemische Umwandlung des Chordagewebes aufzufassen ist, so drängt sich jetzt die Frage auf: Welches ist die Natur des Chordagewebes und wie ist seine Verwandtschaft z um Knorpelgewebe?

Klatsch ist von den neueren Autoren derjenige, welcher, wie wir in einem früheren Kapitel bei Besprechung der Literatur. bereits angeführt haben, in konsequenter Durchführung seiner Ansichten über die Bildung des Chordaknorpels am meisten dazu neigt, eine innige Verwandtschaft zwischen Knorpel und (hordagewebe anzunehmen. Er meint, beide Gewebe könnten atis dem gleichen Stamme eines indifferenten Stützgewebes hervorgegangen sein. Auch Ussow und Ebner haben ähnlich sich ausgesprochen, wenn sie auch die Verwandtschaft beider Gewebe nicht so betont habell. Ich möchte nun auf Grund meiner Befunde bei der Chondrogenese im Chordagewebe der verschiedenen Tierspezies die $K \mathrm{l}$ a a $\mathrm{sch}$ sche Ansicht erweitern und das Chordagewebe als einen Larvalknorpel bezeichnen, d. h. als ein Knorpelgewebe, welches sowohl in der philogenetischen als auch in der ontogenetischen Entwicklungsreihe ein verschiedenartiges Aussehen darbieten kann, je nach den an dasselbe gestellten Ansprüchen. Wie man, um mich dieses etwas trivialen Vergleiches zu bedienen, es einer Kaulquappe auch nicht ansieht, dass sie sich zu einer gewissen Zeit unter Mitwirkung äusserer Verhältnisse zu einem Frosche umwandelt, so verhält es sich auch mit dem Chordagewebe, allerdings mit der Einschränkung, dass die Kaulquappe ein Frosch werden muss, wenn sie nicht vorher zugrunde geht, die Chorda aber nicht Knorpel werden 
muss, sondern auf ihrer früheren Entwicklungsstufe stehen bleiben kann.

Auf der niedersten Stufe sehen wir, wenn wir von den Wirbellosen ('Tunikaten) absehen, beim Amphioxus und den Petromyzonten das Chordagewebe aus zelligen Elementen bestehen, welche selbst in ihrer am weitesten vorgeschrittenen Ditferenzierung, wie in den Zellen des Chordastranges der Petromyzonten, den Krnorpelzellen wenig ahnlich sind. Bei den Kinochenfischen wird im Auftreten der epidermoiden Zellen die Ïhnlichkeit schon grösser und in gewissen Fällen, wie beim Aal und Hecht, schon sehr prägnant. Bei den Urodelen vollends sehen wir dann das Chordagewebe im Chordaknorpel die höchste Ausbildung eil'eichen. Es wird hier ein echtes lyalines Knorpelgewebe gebildet, welches nicht nur wie bei den höheren Wirbeltieren eine provisorische, sondern eine bleibende Einrichtung darstellt und zur Stützfunktion mit beitragen hilft, indem es den Wirbel in seinel Mitte, wo er den lieinsten Querschnitt hat, verstïlit.

Was mu den Chordaknorpel speziell betrifft, so hat bereits Klatsch die plylogenetische Bedeutung des Chordastranges bei Petromyzonten hervorgehoben und die Zellen desselben mit den mittleren Zellen der Amphioxuschorda verglichen. Ich möchte in Erweiterung dieser Anschauung meine Ansicht dahin aussprechen, dass die mittleren Zellen des Amphioxus, der Chordastrang der l'etromyzonten und der Kinochentische, sowie der Chordalinorpel der Urodelen gleichwertige Bildungen darstellen.

Auch komute ich in den verschiedenen Entwicklungsstadien der Chorda der Axolotllarve Befunde konstatieren, welche ich als eine ontogenetische Rekapitulation plyylogenetischer Entwicklungsstufen deuten möchte. Zunächst fiel mir bei Axolotllarven von $1 \frac{1}{2} \mathrm{~cm}$ Länge eine eigentïmliche Anordnung der Chordazellen auf in der Art, dass die Mehrzahl der Zellendoplasmen mit ihrem Kern in der Mitte - in der zentralen Längsachse - der Chorda lag. Ich möchte in diesem Verhalten ein Stadium sehen, wie es beim Amphioxus erst zu spaterer Zeit der ontogenetischen Entwicklung in den mittleren Zellen der Chorda zum Ausdruck kommt. Weiter konnte ich konstatieren, dass einzelne dieser Zellen eine eigentümliche Beschaffenleit ihres Protoplasmas darboten, welche sich dadurch auszeichnete, 
dass es sich mit Farbstoffen furbte, welche Schleim und Chondromukoid färben. So fürbte es sich z. B. mit Kresylviolett rot, während das Protoplasma der übrigen Zellen ungefärbt blieb. Bei etwas grösseren Larven von $21 / 2 \mathrm{~cm}$ kommt es nun schon zu einer Bildung; wie wir sie als Einleitung zur Knorpelbildung beschrieben haben und durch Verdickung der Zellmembran und Ausbildung eines Fadennetzes in den Vakuolen charakterisiert ist. Ich möchte in dieser Bildung ein Stadium erblicken, wie es im Chordastrang der Petromyzonten und Knochenfische in Erscheinung tritt.

Dem Cesagten zufolge wiilde die alte Köllikersche Ansicht, welche die chorda für einen Zellenknorpel erklärte, in gewissem Simne zu liecht bestehen bleiben. Köolliker bezeichnete dabei den Zellenknorpel als einen Knorpel ohne Zwischensubstanz. Die Zwischensubstanz wird nach ihm nur durch die versclimolzenen Membranen der Kinorpelzellen dargestellt, letztere sind aber Produlte der Zellen (Protoblasten).

Icl habe num den Ausdruck Larvalknorpel gewahlt, weil ein Zellknorpel im Köllikerschen sinne noch nicht als echter Knorpel zu bezeichnen ist, denn zum Begrift des Knorpelgewebes gehört doch die Eigenschaft des Gewebes, in seiner Grundsubstanz vorübergehend oder dauernd Chondromukoid auszuscheiden. Auch möchte ich annelımen, dass bei einem solchen Gewebe, wie die Chorda, zwischen den verschmolzenen Membranen (Exoplasmen) doch noch eine, wenn auch minimale und nicht notwendigerweise nachweisbare Menge von verbindender Kittsubstanz vorhanden ist. Sonst würde sich der innige Zusammenhang der Jembranen nicht erklären lassen.

Der Begriff des Köllikerschen Zellknorpels dürfte auch gleichbedentend sein mit dem, was man unter Vorknorpel versteht. Diese Benennung würde nun wohl auch vielen Eigenschaften des Chordagewebes gerecht werden und hat auch Studnička schon früher den Ausspruch getan, dass das embryonale Chordagewebe einem Vorknorpel fast identisch ist. Immerhin aber habe ich auch von dieser Bezeichnung Abstand genommen, weil man unter Vorknorpel sehr verschiedene Dinge begreift: einmal ein bestimmtes Stadium der Chondrogenese: das Chondroblastem und ferner den bleibenden Vorknorpel, das sogenannte blasige Stützgewebe (Schaffer) und seine Modifikationen. 
Auch wird für den Vorknorpel als charakteristisch die homogene hyaline Beschaffenheit des Zellprotoplasmas und die Acidophilität der Interzellularsubstanz angesehen, was für die Chorda doch nur unter gewissen Verhältnissen zutreffen würde. Ich halte es für zweckmässig, den Namen "Vorknorpel" nur für das Chondroblastem $\mathrm{zu}$ reservieren.

Ich komme nunmehr auf die Anschaunngen derjenigen Forscher zu sprechen, welche die Chorda für ein Epithelgewebe halten und in der Art der Verbindung der Chordatellen untereinander durch In terzell u la rb ü cken das wichtigste

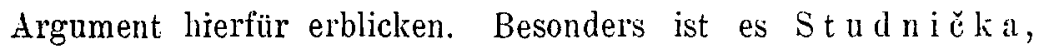
welcher diesen Standpunkt einnimmt. Er selbst hat diese Verbindung hauptsächlich bei Teleostieru gesehen und gibt zu, dass bei Amphibien ihr Nachweis nur in ganz seltenen Fallen ihm gelungen sei. Obwohl ich nun meine Aufmerksamkeit anf diesen Punkt häutig gerichtet habe, konnte ich mich niemals von der Anwesenheit solcher Verbindungen bei Amplibien iberzeugen, selbst nicht bei ganz jungen Tieren. Was ihr Vorkommen bei Knochenfischen betrifft, z. B beim Hecht, so sehen allerdings, wie auch Ebner dies zuerst schon hervorgehoben hat, die Verbindungen der Chordazellen den Protoplasmafasern von Epithelzellen der Epidermis ausserordentlich alnnlich, weshalb die Chordazellen auch als epidermoide Zellen von Ebner bezeichnet wurden. Ich sehe indes in der Beschaffenheit dieser \%ellen keinen (irund, sie als Epithelzellen aufzufassen. Die Mächtigkeit und der mregelmässige Verlauf der Fasern spricht eher gegen ihre epitheliale Beschaffenheit. Ausserdem konnte ich im Intervertebralknorpel von jungen Axolotl- und Tritonenlarven Ḱnorpelzellen finden, welche in ihrem Aussehen und ihrer Verbindungsweise eine grosse Ïhnlichkeit mit den epidermoiden Zellen der Hechtchorda hatten. Wie schon vor vielen Jahren von Flesch, Frommann, o. Hertwig $u$. a. beschrieben worden ist, komme neben solche protoplasmatische Fasern, welche von den Zellen aus an die Kapsel und noch weiter in die Grundsubstanz verlaufen können, gerade auch beim Knorpelgewebe vor. Auch E. Retterer hat bereits früher und ganz neuerdings ein im wesentlichen radiür verlaufendes Faserwerk zwischen Kern und dell von den peripheren Zellpartien gebildeten Kapseln als charakteristisch für das Kinorpelgewebe nachgewiesen. Schliesslich möchte ich auch au den 
Schadelknorpel der Cephalopoden erinnern, wo solche die Grundsubstanz durchziehenden Protoplasmafaserzüge schon seit langem bekannt sind.

Die von Studnička im Anfang der Chordaentwicklung beobachtete Bildung von interzellulären Lücken im Synzytium der noch nicht getrennten Zellen habe ich auch bei der Bildung des Chordaknorpels im Protoplasma, welches aus sich teilender (hordaepithelzellen hervorgegangen war, gesehen (Fig. 3, Taf. IV). Bei meiner Beobachtung handelte es sich also um Zellen, welche zu Knorpelzellen wurden. Ich möchte die Bildung der Interzellularlücken, welche nach Studnička der Bildung der Protoplasmafasern vorausgeht, in eine Linie mit der Vakuolenbildung der differenzierten Chordazellen stellen. Das Protoplasma der embryonalen Chordazellen hat eben die Tendenz, mit Flüssigkeit erfüllte Hohlrĭume za bilden und kann, wie wir dies besonders bei der Chorda der 'Teleostier sehen, die Bildung der Vakuolen bezgl. des Ortes, der Zahl, der Grösse eine sehr rerschiedene sein. Ich sehe deshalb die Bildung von Interzellularräumen und Interzellularbrücken nur als eine zufälige an, weshalb sie auch nicht als charakteristisch für die epitheliale Natur des Chordagewebes anzusehen ist. Für gewöhnlich und bei den Urodelen in der Regel grenzen die Nembranen der Chordazellen glatt aneinandor und werden, wie man annelimen muss, durch eine geringe, went auch nicht nachweisbare Menge von Kittsubstanz oder nach Waldeyerscher Auffassung von Interzellularsubstanz zusammengehalten. Die miteinander verschmolzenen Membranen oder Exoplasmen der Chordazellen kann man als der (ilund- resp. Kapselsubstanz des Knorpelgewebes gleichwertig erachten.

Auch der von den Anhängern der epithelialen Beschatfenheit der Chorda erhobene Einwand, dass die Chorda entsprechend den Untersuchungen von Retzi us kein Chondrin enthalte und deshalb dem Knorpelgewebe nicht zugerechnet werden dürfe, bedarf nach der von mir gegebenen Bezeichnung der Chorda als eines Larvalknorpels eigentlich keiner Widerlegung. Das Chondrin erscheint eben nur zu einer bestimmten Zeit, sobald die Chorda die Stufe ihrer höchstmöglichen Entwicklungsfähigkeit, wie sie sich in der Bildung des Chordaknorpels ausdrückt, erreicht hat. 
Ich glaube, dass nach den von mir gegebenen Darlegungen die Definition der Chorda als eines Larvalknorpels die so sehr wechselnde Beschaffenheit derselben bei den verschiedenen Tierklassen und deren verschiedenen Entwicklungsstufen am besten präzisielt.

Die aus der vorstehenden Arbeit sich ergebenden Resultate möchte ich in folgenden Sätzen zusammenfassen:

1. Der Chordaknorpel der Urodelen entsteht sowohl aus den Chordaepithelzellen ais aus den vakuolisierten Chordazellen. Der erste Beginn der Bildung des Chordaknorpels gibt sich beim Axolotl durch eine Verdickung der Zellmembranen und durch Bildung eines Netzwerkes im Imnern der vakuolisierten Zellen des Vertebralteils der Chorda zu erkennen. Erst später beginnt das Chordaepithel sich an der Chondrogenese zu beteiligen.

Die Chordaepithelzellen liefern nur die peripheren Partien des Chordaknorpels, indem sie ein protoplasmatisch faseriges Netz, in ilurer Umgebung gleichsam eine Verbreiterung der Faserscheide, ausarbeiten und sich durch '́eilung vermehren.

Das Endoplasma der vakuolisierten Zellen der Chorda zeigt dagegen keine oder nur selten Vermehrungsvorgänge. Unter seinem Eintluss und dem der ausgewanderten rom Chordaepithel abstammenden Zellen kommt es durch Ausscheidung einer kolloiden Substanz in die Vakuolenthüssigkeit und die Flüssigkeit der übrigen Spaltrüume der Chorda zu einer Gelatinierung derselben, wobei sich Tropfen und Netze bilden, welche die Grundlage für die chondromukoide Metamorphose abgeben.

2. Die Chorda, obwohl sie ein entodermales Gebilde und aus dem Epithel entstanden ist, ist dennoch nicht dem Epithelgewebe zuzurechnen, wie dies heute vielfach geschieht. Sie hat mit demselben nichts gemein. Thre zuweilen vorhandene Ähnlichkeit mit dem Epithelgewebe ist nur eine rein äusserliche und zufällige. Die Chorda zeigt vielmehr durch ihr Verhalten bei der Bildung des Chordaknorpels, dass sie in inniger Beziehung zu dem aus dem Mesoderm hervorgegangenen Knorpelgewebe steht. Ich möchte die Chorda zur Gruppe der chondroiden Gewebe zühlen, in welche auch das vesikuläre Stützgewebe (Schaffer) in der Achillessehne des Frosches und in verschiedenen Knorpeln von Myxine und Petromyzon einzureihen 
ist. Innerhalb dieser Gewebsgruppe nimmt die Chorda jedoch eine besondere Stellung als ein Gewebe sui generis ein. Wenn auch das Aussehen der Chorda auf den verschiedenen onto- und phylogenetischen Entwicklungsstufen ein sehr verschiedenes ist, so kann doch ihre Umwandlung in echtes Knorpelgewebe oder demselben nahestehendes Gewebe durch eine einfache chemischstrukturelle Metaplasie erfolgen, sobald durch die an das. Gewebe gestellten funktionellen Ansprüche eine solche Umwandlung zweckmässig und notwendig ist. Aus diesem Grunde ist die Bezeichnung der Chorda als Larvalknorpel wohl eine berechtigte.

Am Schlusse dieser Arbeit erlaube ich mir, Herrn Geheimrat Hertwig, welcher mir die Anregung zu derselben gab, sowie seinen Assistenten, Herrn I'rof. Kr ra u se und Prof. Poll für das der Arbeit entgegengebrachte freundliche Interesse und die. Unterstützung durch Material meinen aufrichtigen Dank auszusprechen.

Charlottenburg, im Juni 1908.

\section{Literaturverzeichnis.}

1. Barfurth, J.: Die Eirscheinungen der Regeneration. Handbuch der vergleichenden und experimentellen Entwicklungslehre der Wirbeltiere. Herausgegeben von O. Hertwig, 7. Lief., Jena 1903, S. 95.

2. Derselbe: Zur Regeneration der Gewebe. Arch. f. mikrosk. Anatomie, Bd. 37.

3. Bïtsehli, 0.: Untersuchungen der mikroskopischen Schäume und Protoplasma. Leipzig 1892.

4. Derselbe: Strukturen künstlicher und natürlicher quellbarer Körper, Verh. med.-nat. Ges., Heidelberg 1895.

5. Derselbe: Die quellbaren Körper, Abh. Gött. Ges., 1896.

6. v. Ebner, V.: Wirbel der Knochenfische. Sitzungsbericht der Wiener Akademie der Wissenschaft, Bd. CV, Abt. III, 1896.

7. Field, H. H.: Bemerkungen über die Entwicklung der Wirbelsäule bei den Amphibien. Morph. Jahrb., 22. Bd., Leipzig 1895.

8. Flesch, M.: Untersuchungen über die Grundsubstanz des hyalinen Knorpels. Würzburg 1880.

9. Fraisse, P.: Die Regeneration von Geweben und Organen bei den Wirbeltieren bes. Amphibien und Reptilien, Kassel und Berlin 1885.

10. Frommann, C.: Über die Struktur der Knorpelzellen von Salamandra maculata. Sitz.-Ber. d. Jenasch. Ges., 24. Jan. 1879, S. 17. 
11. Gadow: On the evulution of the vertebral column of Amphibia and Amniota. Philos. Transact. of the Royal Soc. of London. Vol. CLXXXVII. 1896 .

12. Gegenbaur, C.: L̈ber Bau und Entwicklung der Wirbelsäule bei Amphibien. Abhandl. ter Naturforsch. Ges. zu Halle, Bd. VI, 1861.

13. Derselbe: Untersuclungen zur vergleichenden Anatomie der Wirbeltiere bei Amphibien und Reptilien. Leipzig 1862.

14. Derselhe: Vergl. Anatomie der Wirbeltiere, Bd. I, Leipzig 1898, S. 241.

15. Götte, A.: Entwicklungsgeschichte der Unke, Leipzig 1875. S. 361, $367-371,377$ usw.

16. Gurwitsch, A.: Morphologie und Biologie der Zelle. Jena 1904, S. 13 u. f.

17. Hansen, F. C. C.: Lntersuchungen iiber die Gruppe der Bindesubstanzen und Hyalinknorpel. Anatom. Hefte v. Merkel u. Bonnet, Bd. 27, Heft 81 .

18. Derselbe: Über die Genese einiger Bindegewebsgrundsubstanzen. Anat. Anz., Bl. 16, 1899. Nr. 17 und 18.

19. Hardy: On the Structure of Cell-Protoplasm. Journ. Physiolog, 1899.

20. Hasse, C.: Die Entwicklung der Wirbelsiiule von Triton tacniat. Zeitschr. f. wiss. Zool., BI. LIII, Suppl. 1892.

21. Derselle: Das natürl. System der Elasmobranchier. Jena, Fischer.

22. Hertwig, O.: Über die Entwicklmg und den Balu des elastischen Gewehes im Netzknorpel. M. Sultzes Arch., Bd. IX.

23. Derselle: Die Zelle und die Gewebe. Bd. I, 1893, S. 127

24. Derselhe: T,ehrbuch der Entwicklungsgeschichte. כ. Aufl., 1896, S. כ50).

25. Kapelkin, W.: Zur Frage iiber die Entwicklung des axialen Skeletts der Amphibien. Bull. Soc. des Naturalistes, Moscon, Tom. XIV, 1900.

26. Kla atsch, H.: Beitr. zur vgl. Anatom. d. Wirbelsiule. I. Über den Urzustand der Fischwirbelsiunle. Morphol. Jahrb., Bd. 19, 1893.

27. Derselbe: Beitr. zur rgl. Anatom. d. Wirbelsüule. III. Morphol. Jahrb., Bd. XXII.

28. Derselbe: Über die Chorda und die Chordascheiden der Amphibien. Verhandl. d. Anatom. Ces., 11. Versamml., 1897.

29. v. Källiker, A.: Z̈ber las Ende der Wirbelsünle der Ganoiden und einiger Teleostier. Leipzig 1860.

30. Derselbe: Entwicklungsgeschichte des Mienschen und der höheren Tiere. Leipzig, II. Aufl., 1879, S. \pm 01, S. 408.

31. Derselbe: Handbuch der Gewebelehre des Menschen, I. Bd., Leipzig, 1889

32. Lwnff. B.: Vergl. Anatom. Studien uber die Chorda und die Chordascheide. Bull. de la Société Imperial-Naturalistes de Moscou, Nr. 2, 1887.

33. Müller, W.: Über den Bau der Chorda dorsalis. Beobachtungen des Pathol. Instituts in Jena. I. Jenasche Zeitschr. f. Mediz. u. Naturwissenschaften. Bd. VI, 1871.

34. Nus baum, J. und Sidoriak, S.: Beiträge zur Kenntnis der Regenerationsvorgänge etc. Arch. f. Entwickl. mechan. von Roux, Bd. X, 1900. 
3o. Rauber, A: Luchrbuch der Anatomic des Menschen, 5. Aufl., 1897, Bd. I, S. $79,97,336$.

36. Rutterer, E.: Evolution du cartilage transitoire. Journ. de I'Anatomie et de la Physiologie, I, 36, 1900, p. 467.

37. Derselbe: De la Structure reticulée de la cellulc cartilageneuse. Comptes rendus de la Société de Biolıg., Tom. 63, 1907, 2. Teil, S. 782.

38. Retzius: Einige Buiträge zur Histologie und Histochemie der Chorda dorsalis. Arch. f. Anat. u. Plyysiol., 1881, S. 89, 108.

39. Ribbert, H.: Uber Veränderungen transplantierter Gewebe. Arch. f. Entwicklungsmochan. v. Ruux, Bd. VI, 1898.

40. Schaffer. J.: Zur Kenntnis des histologischen und anatomischen Baues von Ammocortes. Anat. Anz.; Bd. X, 1895, S. 708.

41. Dersclbe: Über das knorpelige Skelett von Ammocoetes branchialis nebst Bemerkungen ïber das Knorpelgewebe im Allgem. Zeitschı'. f. wissensch. Zoul., Bd. 61, 1896, S. 639, Anm.

42. Derselbe: Bemerkungen ïber die Histologic und Histogenese des Knorpels der Cylilostome. Arcl. f. mikr. Anat., Bd. 50, 1897.

43. Derselbe: Grunlsubstan\%, Interzellularsubstanz und Kittsubstanz. Anat. Anz., Bd. XIX, 1901, S. 95.

44. Derselbe: Cber den feincren Ban und dis Entwicklung des Knorpelgewehes usw. Zeitschr. f. wissensch. Zool., Bd. 70, 1901, S. 147.

45. Dersube: ت̈ber den feineren Bate und die Entwicklung des Knorpelgewroles usw. Zeitschr. f. wissensch. Zool., Bł. 80, 1905), S. 190 u. ff.

46. Schauinsland, H.: Dic Entwicklung der Wirbelsäule nebst Rippenund Bristbein. Handb. d. Entwirlilungslehre, herausgegeb. v. O. He rtwig, Bd. III, 2. Teil.

47. Sehmidt. V.: Das Schwanzende der Chorda dorsalis bei den Wirbeltieren. Anat. Heftre, Bil. II, I. Abt., 1893.

48. Schnoid(r, A.: Be-itl. zur verol. Anat. w. Entwicklungslehre der Wirbeltiere. Burlin 1879.

49. Schneider, Camillo: Histologic dex Tiere. Jona, G. Fischer, 1902, S. 793 .

50. Strasser, H.: Zur Entwicklung des Extremitïtenlnorpels bei Salamander und Triton. Horphol. Jahrib. Bd. V, 1879, pas. 240 ff.

51. Studnicka. F, K.: Uber das Gewebe der Chorta dorsalis und den sogenannten Chordaknorpel. Sitzungsber. der Kgl. Böhm. Gesellsch. der Wissensehaften, mathemat.-naturwissenschaftl. Klasse, 1897.

52. Derselbe: Histologische und histogenetische Lintersuchungen über das Knorpel-, Vorknorpel- und Chordagewebe. Anat. Hefte von Merkel und Bonnet, Bd. XXI (Heft LXVI u. LXVII).

53. Ussow: S.: Zur Anatomie und Entwicklungsgeschichte der Teleostier. Bull. de la Société Inup. des Naturalistes de Moscou. Année 1900, Nouv. Série, Tom. XIV, Moscou 1900.

54. Waldeyer, W.: Kitt- und Grundsubstanz, Epithel und Endothel. Arch. f. mikr. Anat., Bd. 57, 1901. 
55. Wolters, Max: Zur Kenntnis der Grundsubstanz und der Saftbalnen des Knorpels. Arch. f. mikr. Anat., Bd. 37 u. 38, 1891.

56. Zykoff, W.: Über das Verhältnis des Knorpels zur Chorda bei Siredon pisciformis. Bull. de la Société Impérial de Moscou. Anné 1893, Nouv. Serie, Tom. VII, Moscou 1894.

\section{Erklärung der Abbildungen auf Tafel IV-VI.}

Fig. 1. Axolotl von $3,6 \mathrm{~cm}$ Länge. Frontalschnitt durch die mittlete Rumpfpartie. I. Stadium der Bildung des Chordaknorpels. Auf den Vertebralteil der Chorda beschränkte Veränderung der vakuolisierten Zellen: Verdickung der Nembranen und Netzbildung innerhalb der Valkuolen. Bleistiftzeichnung. Hartnack Okular I, Obj. 3, Tubus zur Hälfte ausgezogen mit Verschiebung des Prüparates gezeichnet. 1 $1 \frac{1}{3}$ Gesichtsfeld. Fixierung: Pikrin-SullimatEssigsäure. Färbung: Boraxkarmin, Bismarckbraun, Lichtgrün.

Fig. 2. Ein Teil desselben Präparates wie Fig. 1 bei stäkerer Vergrösserung: Leitz Öl-Imm. 1/12, Okul. 1, Tub. $160 \mathrm{~mm}$. Wan sieht, wie einige Fasern aus aneinander gereilten Tröpfchen bestchen und mit Knorpelfarbstoffen sich fürben.

Fig. 3. Axolotl von $4 \mathrm{~cm}$ Länge. Teil eines Querschnittes der obcren Rumpfgegend. Erste Bildung des Chordaknorpels im Bcreich des Chordaepithels. Vermehrung der Chordaepithelzellen, welche gleichzeitig von der Faserscheide abrücken. Bildung von protoplasmatischen Netzen und kleinen Vakuolen. In der Peripherie einer Zelle sieht man reihenförmig angeordnete kleinste Vakuolen. welche interzellalären Verbindungen, wie sie zwischen Epithelzellen vorkommen, ähnlich sehen. Leitz Öl-Imm. ${ }^{1 / 12}$, Ok. 3, Tuljus $160 \mathrm{~mm}$ Färbung: Hämalaun, Kresylviolett.

Fig. 4. Axolotl von $4 \mathrm{~cm}$ Länge. Teil eines Querschnittes durch die Chorda der oberen Rumpfpartie. Beginn der Knorpelbildung in der Peripherie der Chorda. Das Epithel ist in der Faserscheide durch Netz- und Vakuolenbildung abgeruickt und zeigt geringe Beteiligung. Bildung zahlreicher, meist in Gruppen angeordneter kleiner Vakuolen. Oft folgen sich die Vakuolen reihenförmig in zunehmender Grösse. In einigen Vakuolen chondromukoide Netze und Tröpfchen. Einzelne sehr grosse Vakuolen mit fein- oder weitmaschigem Netzwerk. Dazwischen breite Septa, welche zum Teil ebenfalls faserige, netzförmige Beschaffenheit und hie und $d a$ auch. chondromukoide Fasern zeigen. Leitz Öl-Imm. 1/12, Ok. 1, Tub. $160 \mathrm{~mm}$. Fixierung: Pikrin-Sublimat-Essigsäure. Färbung: Hämalaun, Kresylviolett.

Fig. 5. Gleiches Objekt wie in Fig. 4. Frontalschnitt durch die Schädelchorda (Occipitalgegend). Frühes Stadium der Knorpel- 
bildung in der Chorda. Das Epithel hat sich hier stärker beteiligt. Man sieht zahlreiche in der bereits verknorpelten Faserscheide eingeschlossene Knorpelzellen: Abkömmlinge der Epithelzellen der Chorda. Hie und da hat sich schon eine Kapsel gebildet. Daneben auch in die Grundsubstanz eingeschlossene Protoplasmamassen. Metaplastische chondromukoide Prozesse an den vakuolisierten Zellen in Form von Netzen und Tröpfchen, letztere besonders am Rande der Valiuolen. Interessante albuminoide Netzbildung und Bildung sekundärer Vaknolen. Leitz Öl-Imm. ${ }^{1 / 12}$, Ok. 3, Tub. $160 \mathrm{~mm}$. Fixierung und Färbung wie bei Fig. 5 .

Fig. 6. Axolotl von $3,6 \mathrm{~cm}$ Länge. Frontalschnitt durch die Chorda der oberen Rumpfpartie. Chordaknorpel teils vom Chordaepithel gebildet, teils durch Metapiasie valiuolisierter Zellen entstanden. In der Umgebung des Chordaknorpels haben sich ausgewanderte, durch Teilung aus Chordaepithelzellen hervorgegangene Zellen angehäuft, welche zum Teil in die Grundsubstanz des Chordaknorpels eingeschlossen werden. Starkes Vordrängen von seiten des Knorpels des oberen Bogens gegen die Elastica ext. Letztere trotzdem iuberall intakt. Faserscheide an manchen Stellen schon betrïchtlich in die Chordaknorpelbildung einbezogen. Lueitz Öl-Imm. 1/12, Ok. 3, Tub. $160 \mathrm{~mm}$ mit Verschiebung gezcichnet. Fixierung: Pikr.-Subl-Esssigs. Fürbung: Hämalaun, Kresylviolett.

Fig. 7. Triton cristatus von $5,5 \mathrm{~mm}$ Lünge. Frontalschnitt durch die Chorda der unteren Rumpfpartie. Chordaknorpel mit Chordastrang, letzterer mit Eosin leuchtend rot gefärbt. Auch in den Intervertebralteilen der Chorda sieht man an zwei Stellen insuläre Knorpelbildungen. Auch haben sich hier einige strangartig verdickte Chordafaserzüge mit Eosin rot gefürbt. Leitz Obj. 3, Ok. 3, Tub. ausgezogen. Fixierung: Pikr.-Subl.-Essigs. Fïrbung: Hämalaun, Eosin.

Fig. 8. Axolotl von zirka $8 \mathrm{~cm}$ Lünge. Frontalschnitt von der mittleren Schwanzpartie nahe dem Chordastab. Chordaknorpel von noch jüngerer Entstehung. Die Grundsubstanz von zahlreichen Chordaresten durchzogen in Form von feineren und gröberen Faserzügen. Die Knorpelzellen haben oft weite, unregeimässig geformte Kapselhöhlen und enthalten zuweilen Protoplasmamassen ohne Kern. Öfters ist die Kapsel noch nicht gebildet oder nur angedeutet. Mit dem Chordaknorpel ist noch ein proximalwärts gelegener Chordastrang in Verbindung, in welchem es teilweise schon zu einer chondromukoiden Metamorphose gekommen ist. Derselben geht eine albuminoide, acidophile Beschaffenheit der Strangpartien voraus. Leitz Obj. 6, Ok. 3 mit Verschiebung gezeichnet $\left(1^{1 / 4} \mathrm{Ge}\right.$ sichtsfeld). Fixiernng: Pikr.-Subl.-Essigs, Färbung: Bismarckbraun, Lichtgrün.

Fig. 9. Axolotl von zirka $8 \mathrm{~cm}$ Länge. Frontalschnitt. Eine in der Nähe der vorigen Figur, aber mehr proximalwärts gelegene Stelle. Kn orp elige

Archir f. mikrosk. Anat. Bd. 78 . 
Metaplasie der vakuolisierten Chordazellen, chondromukoide Umwandlung des feinen netzförmigen Valkuoleninhalts, welcher vielfach noch albuminoide, acidophil gefärbte Partien zeigt. Mitunter sieht man auch stark gewundene Fasern. herrührend von den Wänden früherer ausgedehnter Vakuolen, ferner breitere membranöse Bildungen. In vielen Vaknolen sieht man noch das früheste Stadium der Chondrogenese: Feines fädiges Netzwerk, welches zum Teil chondromukoide Fürbung angenommen hat. Vom Epithel ist wenig zu sehen. Auch die Faserscheide ist hier, wie gewöhnlich in der Schwanzpartie älterer Larven nicht ausgebildet. Leitz Öl-Imm. 1/12, Ok. 1, Tub. $160 \mathrm{~mm}$. Fixierung: Pikrin-Subl.Essigs. Färbung: Bismarckbraun, Lichtgrün.

Fig. 10. Chordaknorpel eines $12 \mathrm{~cm}$ langen Axolotls. Querschnitt durch die mittlere Rumpfgegend. Der Chordaknorpel reicht bis an dic noch gut erhaltene, zum Teil etwas verdünnte Elastica ext. Von der Faserscheide ist kaum noch etwas zu bemerken. Die Knorpelzellen liegen in einer teils homogenen, teils feinfaserigen Grundsubstanz. Einige Knorpelzellen sind gut ausgebildet. Viele Knorpelkapseln zeigen einen nur faserigen oder faserig-protoplasmatischen, mit Tröpfchen besetzten Inhalt. Die Kapselwände sind vielfuch konzentrisch geschichtet und von besonders stark ausgeprägter Knorpelfärbung. Man sieht auch einige nur wenig sichtbare, schattenartigre Zellen, welche sich in Grundsubstanz umwandeln (,verdämmernde Zellen ${ }^{4}$, Schaffer). Etwas seitlich von der Mitte die mit Eosin intensiv rot gefärbten Reste des Chordastranges. Nach aussen von der Elastica ext. die noch in Ossifilation begriffene Fiserknochenschicht. Leitz Obj. 6, Ok. 3 mit Verschiebung gezeichnet. Fixierung Pikr.-Subl.-Essigs. Färbung: Hämatoxylin, Eosin.

Fig. 11. Regeneriertes Schwanzende eines Axolotlembryos. Schwanz im Ei amputiert, getötet 9 Tage später. Das Regenerat, welches die ersten Anfünge der Chordastabbildung zeigt, besteht aus Chordazellen, welche embryonalen Charakter tragen und lebhafte Kernteilung zeigen. Auch sieht man im synzytialen Gewebe Neigung zur Vakuolenbildung. Mehrere kleinere und eine grössere Vakuole. In den angrenzenden Zellen der alten Chorda sind metaplastische Prozessezu bemerken. Netz- und Wabenbildung sowie geringe Kernvermehrung. Beginnende chondromukoide Färbung einzelner Chordapartien. Auch die nengebildete Chordascheide, welche schmal und aus schleimhaltigem Bindegewebe besteht, hat ebensolche Färbung angenommen. Man sieht am Ende der Chorda einen zweimaligen Abschluss durch eine solche Faserbildung. Distalwärts von derselben geht der Chordastab allmählich in ein mehr lockeres, zellig retikuläres Gewebe über und reicht dort dicht an die Epidermis. Leitz Öl-Imm. 1/12, Ok. 3, Tub. $160 \mathrm{~mm}$. Fixierung: Pikr.-Subl.-Essigs. Färbung: Hämalaun, Kresylviolett.

Fig. 12. Forellenembryo von $3 \mathrm{~cm}$ Länge. Vier Wochen alte Kontinuitätstrennung der Chorda. Starke Hervordrängung und Aus- 
dehnung des zentralen Chordastumpfes. Weitere Beschreibung im Text (Seite 91 und 92). Leitz Obj. 3, Ok. 3, Tub. ausgezogen. Fixierung: Pikr.-Subl.-Essigs. Fürbung: Hämatoxylin, van Gieson.

Fig. 13. Vier Wochen altes Transplantat der Chorda rom Axolotl. Das Transplantat hängt an einer kleinen Stelle mit dem intermuskulären Bindegewebe zusammen. Im übrigen ist es von einem Lymphraum umgeben, in welchem einige Endothel- und Lymphzellen suspendiert sind. Das Chordagewebe im Transplantat ist noch an den zahlreichen kleinen Vakuolen zu erkennen, welche teils durch schmälere, teils etwas breitere Protoplasmazüge getrennt sind, in welchen hie und da kleine runde, chromatinreiche Keme liegen. In der Umgebung dieses vakuolenhaltigen Gewebes sielnt man zahlreiche Riesenzellen und als äusserste Schicht ein junges zellenreiches Bindegewebe. Leitz Obj. 3, Ok. 3, Tub. ansgezngen. Fixierung: Carnoy. Fürbung: Hämatoxylin, van Gieson.

Fig. 14. Eine Stelle desselben Präparates bei stïrkerer Vergrösserung. Kleine Vakuolen der transplantierten Chorda von protoplasmareichen Zügen umgeben. In letzteren grosse epitheloide, sowie kleine chromatinreiche Kerne. Leitz Öl.-Imm. 1/12, Ok. 1, Tub, $160 \mathrm{~mm}$.

Fig. 15. Vier Wochen altes Transplantat der Axolotlchorda. Man sieht grüssere und kleine Vakuolen mit fein wabenartigem Inhalt. Dizwischen breite kernhaltige Protoplasmazüge und protoplasmareiche Zellen mit grossen Kernen. Häufige Kernteilungsfiguren. Elastica cxt. erhalten, leicht gerquollen. Von der Faserscheirle ist nichts mehr wahrzunehmen. Das Transplantat ist von intermuskulärem Bindegewebe umgeben. Leitz Öl-Imm. 1/12, Okul. 1, Tub. $160 \mathrm{~mm}$. Fixierung: Carnoy. Fürbung: Hümatoxylin, van Gieson.

Fig. 16. Drei Wochen altes Transplantat der Axolotlchorda in einem nahe der Haut befindlichen Granulationsknopf. Beträchtliche Hümorrhagien in der Umgebung und in Innern des Transplantats. Schollig zerklüftete Faserknochenschicht. Im Innern des Wirbelquerschnittes die stark gefaltete und gequollene Faserscheide. Riesenzellen. Sternförmige Zellen und Spindelzellen. Ein Gefässdurchschnitt $z$ wischen Faserscheide und Knochen. Leitz Obj. 6, Oluul. 3, Tub. eingeschoben. Fixierung: Pikrin-Sublim.-Essigsäure. Färbung: Haematoxylin, van Gieson.

Schematische Figuren, welche häufiger vorkommende oder besonders eigenartige Details des Vakuoleninhalts, sowie der Scheidewände bei der Chondrogenese der Chorda veranschaulichen und in den früheren Abbildungen nicht oder nicht genügend typisch enthalten waren. Die Abbildungen sind von Präparaten einer Schnittserie entnommen, von welcher auch Fig. 5 herrührt. Zeiss Apochromat $2 \mathrm{~mm}$, n. Ap. 1,30, Ok. 2.

Fig. 17-28 inkl. 37. Verschiedene durch Konfluenz von Tröpfchen entstandene Bildungen, welche meist mit Kresylviolett rote Färbung angenommen haben. Fig. 25 zeigt ausserdem nochVakuolen- 
wände mit typischer Bildung von Netzwerk und perlschnurartig aneinandergereihten, kleinsten Vakuolen.

Fig. 29-35. Membranbildungen in den Vakuolen. (Die Membran in der Vakuole von Fig. 34 ist im Druck etwas zu hart und scharflinig herausgekommen). Fig. 32 und 35 kombiniert mit Bildungen, wie sie in Fig. 21 und 23 dargestellt sind. Fig. 31 zeigt in einer Valruole zwei Endoplasmazellen umgeben von einer membranartigen Bildung. Fig. 33. Ringförmige von der Vakuolenwand umgebene Membran, deren eine Hälfte sekundäre Vakuolenbildung zeigt und chondromukoide Färbung angenommen hat. Das freie Innere des membranösen Ringes wird von radienartig ausgespannten Füden durchzogen, welche ebenfalls teilweise sich rot gefärbt haben.

Fig. 38. Gerinnselartige Massen in einer Vakuole, teilweise kleinste Hohlräume enthaltend.

Fig. 36, 39, 40. Verschiedene Faserbildung in der Vakuole, in Fig. 40 in Verbindung mit einem von wenig Protoplasma umgebenen Kern.

Fig. 25, 28, 33, 41-45. Verschiedene Scheidewand- und Zwickelbildungen. 


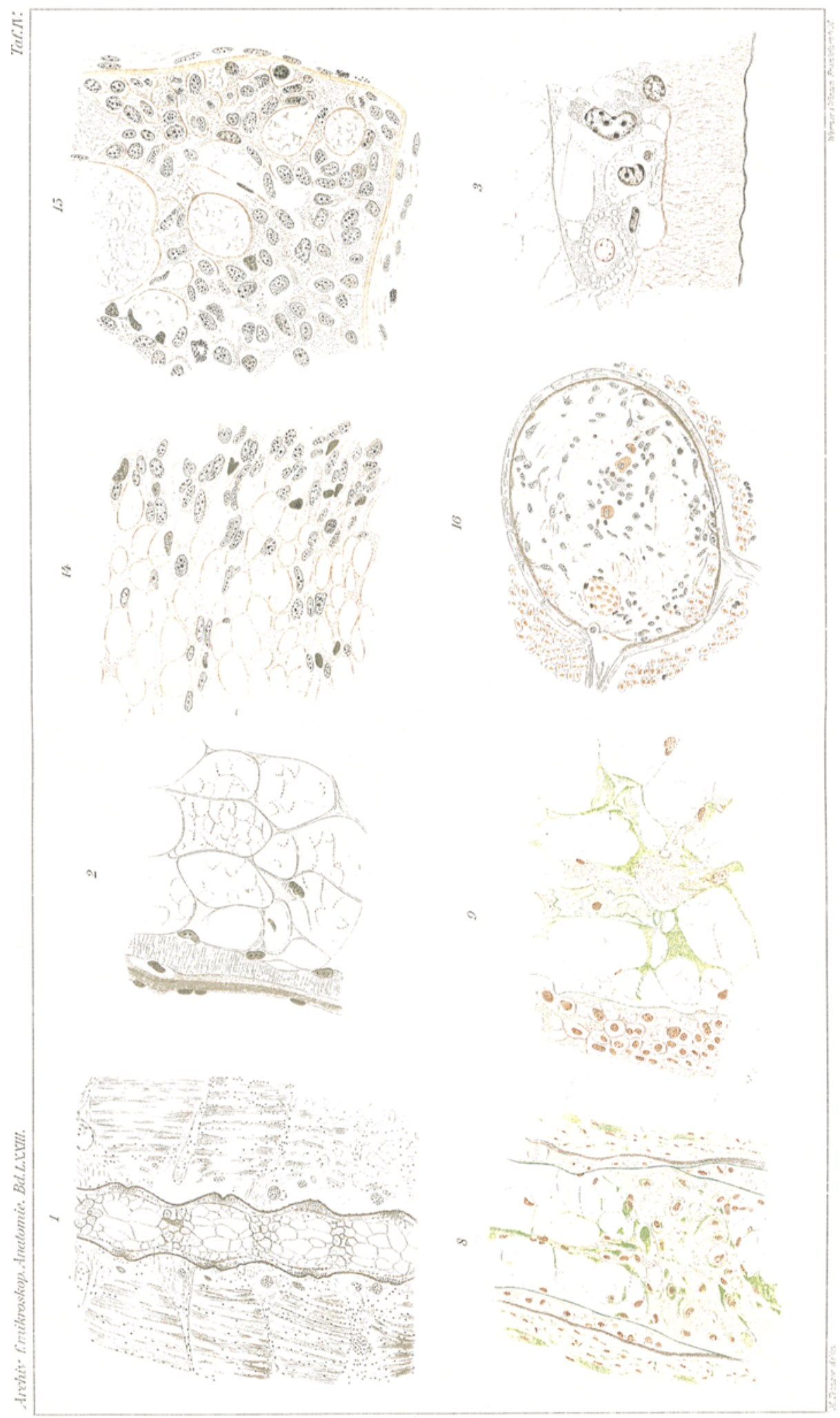




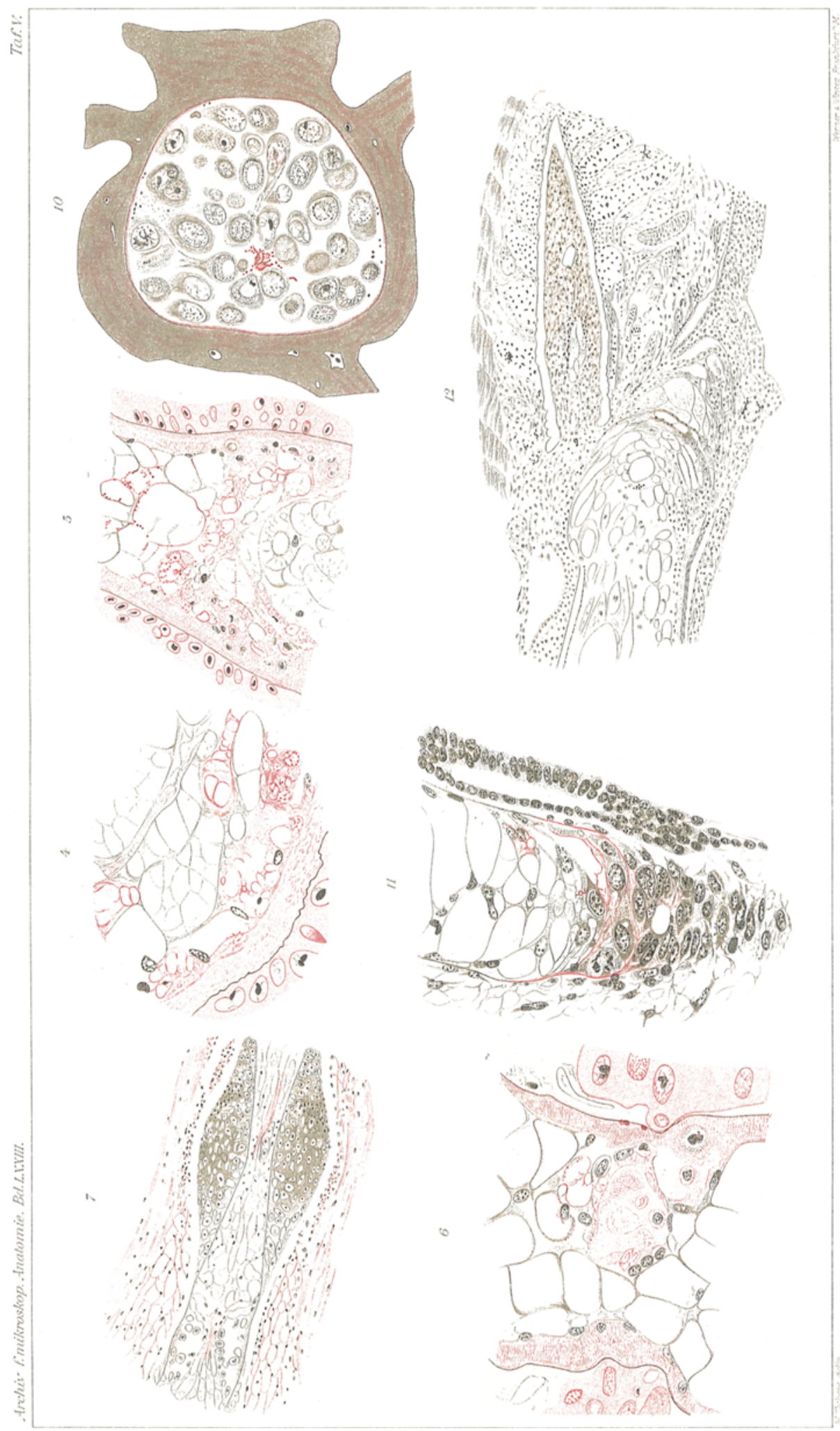




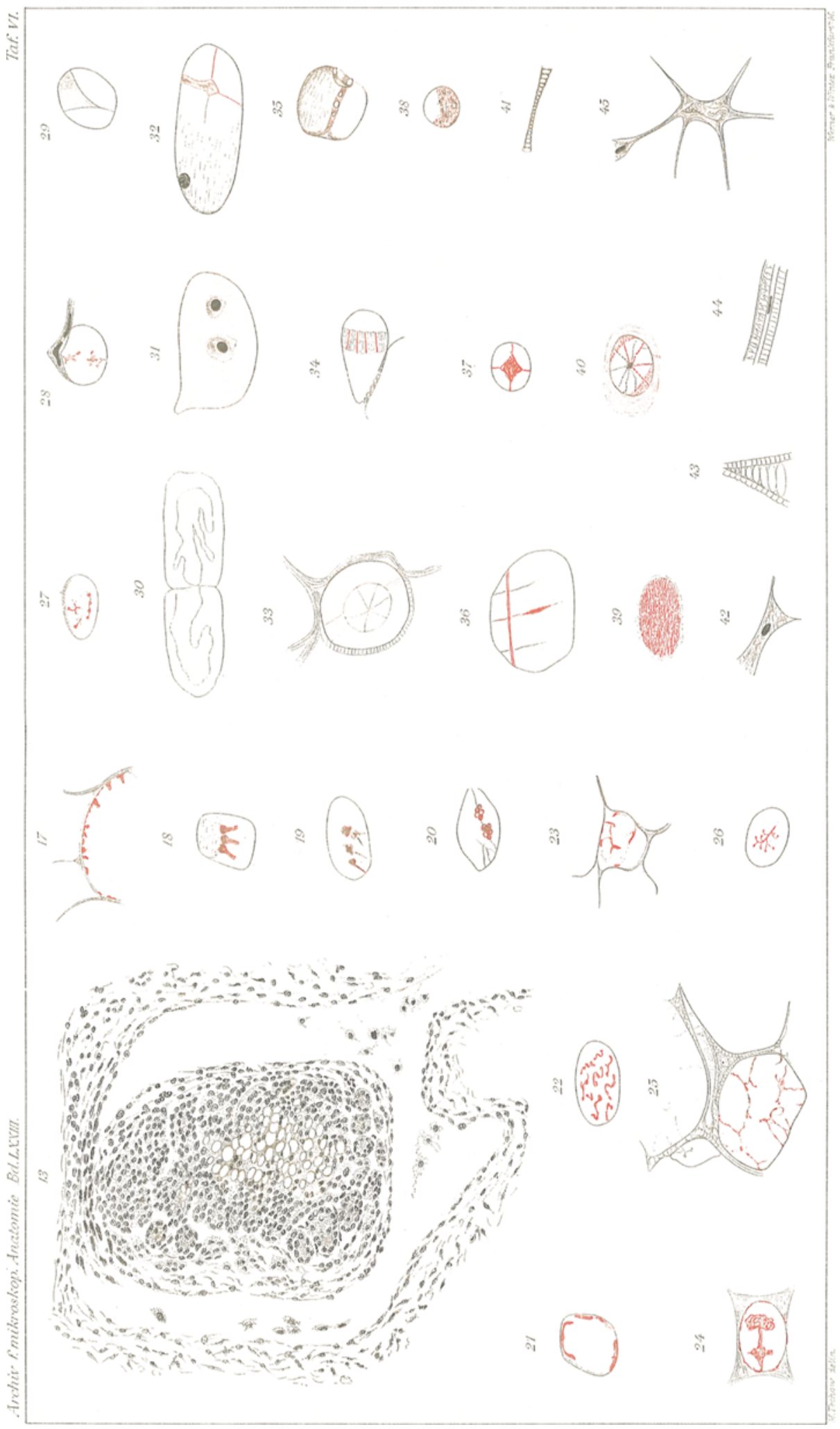

